\title{
Radiation resistance of novel SARS-CoV-2: A Monte Carlo investigation on characterization and determination of gamma-ray attenuation properties
}

H.O. Tekin ( $\square$ tekin765@gmail.com )

University of Sharjah, Sharjah, College of Health Sciences

U. Kara

Suleyman Demirel University

Shams A.M. Issa

Faculty of Science, Al-Azhar University

Ibrahim Inanc

Ondokuz Mayis University

O. Kilicoglu

Vocational School of Health Services, Department of Nuclear Technology and Radiation Protection

Wiam Elshami

University of Sharjah, Sharjah, College of Health Sciences, Medical

Ismail Aslan

University of Health Sciences Turkey

G. Susoy

Istanbul University

Ahmet Arif Kurt

Suleyman Demirel University

Muhsin Konuk

Uskudar University

Mehmet Turan Ayseli

Genetris Consultancy

\section{Research Article}

Keywords: SARS-CoV-2, radiation, radiation attenuation, Monte Carlo simulation

Posted Date: April 24th, 2020

DOI: https://doi.org/10.21203/rs.3.rs-24870/v1 
License: (c) (i) This work is licensed under a Creative Commons Attribution 4.0 International License. Read Full License 


\title{
Radiation resistance of novel SARS-CoV-2: A Monte Carlo investigation on characterization and determination of gamma-ray attenuation properties
}

\author{
H.O. Tekin ${ }^{1}$, U. Kara ${ }^{2}$, Shams A.M. Issa ${ }^{3,4}$, Ibrahim Inanc ${ }^{5,6}$, O. Kilicoglu ${ }^{7,8}$, Wiam Elshami ${ }^{1}$, \\ Ismail Aslan ${ }^{9,10}$, G. Susoy ${ }^{11}$, Ahmet Arif Kurt ${ }^{12}$, Muhsin Konuk ${ }^{13}$, Mehmet Turan Ayseli ${ }^{14,15}$ \\ ${ }^{1}$ University of Sharjah, Sharjah, College of Health Sciences, Medical Diagnostic Imaging Department, 27272, \\ United Arab Emirates \\ ${ }^{2}$ Suleyman Demirel University, Vocational School of Health Services, Medical Imaging Department, Isparta, Turkey \\ ${ }^{3}$ Physics Department, Faculty of Science, Al-Azhar University, Assiut, 71452, Egypt \\ ${ }^{4}$ Department of Physics, Faculty of Science, University of Tabuk, Tabuk, Saudi Arabia \\ ${ }^{5}$ Department of Metallurgical and Materials Engineering, Ondokuz, Mayis University, Kurupelit, 55139, Samsun, Turkey \\ ${ }^{6}$ Department of Nanoscience and Nanotechnology, Ondokuz Mayis University, Kurupelit, 55139, Samsun, Turkey \\ ${ }^{7}$ Uskudar University, Vocational School of Health Services, Department of Nuclear Technology and Radiation Protection, \\ Istanbul, 34672, Turkey \\ ${ }^{8}$ Uskudar University, Medical Radiation Research Center (USMERA), Istanbul 34672, Turkey \\ ${ }^{9}$ University of Health Sciences Turkey, Vocational High School, Department of Pharmacy Services, 34668, Istanbul, Turkey \\ 10 SFA R\&D Laboratories, Teknopark Blv No:3AZ01, 34906, Pendik, Istanbul, Turkey \\ ${ }^{11}$ Istanbul University, Faculty of Science, Department of Physics, 34134, Istanbul, Turkey \\ ${ }^{12}$ Suleyman Demirel University, Vocational School of Health Services, Medical Laboratory Techniques, Isparta, Turkey \\ ${ }^{13}$ Uskudar University, Faculty of Engineering and Natural Sciences, Department of Molecular Biology and Genetics, 34672 \\ Istanbul, Turkey \\ ${ }^{14}$ Genetris Consultancy, 33343, Mersin, Turkey \\ ${ }^{15}$ Ylldız Technical University, Chemical and Metallurgical Engineering Faculty, Department of Food Engineering, 34210, \\ Istanbul, Turkey
}

Corresponding author: tekin765@gmail.com (H.O.Tekin) 


\begin{abstract}
In late December 2019, the new viral pneumonia outbreak was first detected in Wuhan, the largest metropolitan area in China's Hubei province. The 2019-20 coronavirus pandemic is an ongoing pandemic, caused by the severe acute respiratory syndrome-2 (SARS-CoV-2), was named as Coronavirus disease 2019 (COVID-19) by World Health Organization (WHO). It is well known that radiation can cause mutations in bacteria and viruses. Therefore, characterization of the radiation resistance and interaction properties of viruses provides the opportunity in terms of risk assessment and future aspects. In this study, 3 types of viruses (SARS-CoV-CAS Number 58788651-9, Influenza-CAS Number 141368-69-6 and SARS-CoV-2 GlycoProtein 6VSB.) were modeled with the Monte Carlo simulation method (MCNPX version 2.6.0). The vital radiation attenuation properties such as linear attenuation coefficients, energy absorption buildup factors (EABF), exposure buildup factors $(E B F)$, relative dose distributions (RDD) were examined using advanced simulation methods. Moreover, the spike protein of SARS-CoV-2 is modelled from the structures in the Protein Data Bank. As a result of the study, we could say that the most radiation resistance was observed in SARS-CoV when compared with Influenza and Covid-19. It could be one of the reasons for SARS-CoV's resistance to mutation from its outbreak time. On the other hand, Covid19 is more resistant to radiation than Influenza. Therefore it could be expected that Covid-19 would have the similar behaviors against ionizing radiation as Influenza has.
\end{abstract}

Keywords: SARS-CoV-2, radiation, radiation attenuation, Monte Carlo simulation

\title{
1. Introduction
}

In the last twenty years, several viral outbreaks such as "Severe Acute Respiratory Syndrome Coronavirus (SARS-CoV) (2002-2003), H1N1 influenza (2009) and the Middle East Respiratory Syndrome Coronavirus (MERS-CoV) (2012) have been identified in the world [1]. In late December 2019, the new viral pneumonia outbreak was first detected in Wuhan, the largest metropolitan area in China's Hubei province [2]. The 2019-20 coronavirus pandemic is an ongoing pandemic, caused by the severe acute respiratory syndrome-2 (SARS-CoV-2), was named as Coronavirus disease 2019 (COVID-19) by World Health Organization (WHO) [1]. The high mortality is high in older adults 65 years, is unique characteristic of SARS-CoV-2 pandemic [3]. Among these deaths, one of this is quite remarkable because of a newborn death in England. This 
death is unofficially associated with SARS-CoV-2. People with potential exposure to SARS-CoV2 were interviewed with a standardised symptom history like cough, fever, kidney failure, pneumonia and severe acute respiratory syndrome both in older adults and people with any age [4]. This approach has been not particularly useful because some infected people have clinically milder or no symptoms that can be linked transmission of the virus on to others. Recently, it has been shown that infection pediatrics with mild symptoms had positivite test results for fecal transmission [5]. Many scientitst believed that those patients whose not in the "detected pool of infected cases", created new "epidemic hotspots" including different materials (plastic, cardboard, metal) during traveling or working [6]. As the resutls of Cov pandemic, most of countries around the world are stepping up efforts to tackle with COVID-19 based on standard recommendations including covering mouth \& nose when coughing and sneezing, regular hand washing [5]. The literature review showed that term of ionizing radiation is a considerable tool for virus inactivation aims. The term of radiation is the emission or transmission of energy in the form of waves or particles. It is divided into ionizing and non- ionizing radiation based on its energy and causing of biological effect. The main two characteristics of ionising radiation are high energy levels and high penetration through biological and non-biological materials. Drastic increase in the utilization of ionizing radiation in the field of medicine and industry has been noted over the past years $[7,8]$. Therefore, ionising radiation is used in food applications, clinical sterilization, and vaccine preparation. Ionising radiation possessed an ability to inactivate viruses and used for vaccine development as it damages the nucleic acids rather than proteins [9]. However, chemical and physical methods can be used for viruses' inactivation, but ionizing radiation has the advantages of penetration throughout the material. On the other hand, the radiation dose can be measured precisely $[10,11]$. Furthermore, exposure to ionizing radiation has multiple effects on viability and 
further proliferation of the irradiated cell and damage might occur if the exposure dose exceeded the threshold of each specific tissue. Therefore, fractionation is used to deliver the total radiation dose over fractions to allow intracellular repair $[12,13]$ and the live attenuated microorganisms can be used as vaccine materials [13]. Withal, radiation sensitivity is determined by decimal reduction value $\left(D_{10}\right)$ and the shape of the inactivation curve. It is difficult to predict radiation sensitivity but most of the viruses are relatively radiation resistant in a $\mathrm{D}_{10}$ range from 1-10 kGray for gamma photons $\left(\mathrm{Co}^{60}\right)$ [14]. High energy electron and gamma photons are usually used, and in some cases, $\mathrm{x}$ ray and ultraviolet rays are used. However, due to the production of Bremsstrahlung radiation, proteins damage can occur during the process of virus's inactivation using gamma rays $[9,15]$. It's worth to mention that Gamma radiation is an effective way to inactivate influenza. An exposure of 0.65 kiloGrays (kGy) leads to complete inactivation of the virus, and disruption of protein and haemagglutinating activity occurred at exposure to higher than 200KGy [16,17]. Additionally, the effect on the proteins can be reduced with the use of electron, but electron irradiation is less penetrating than gamma rays [15]. In contrast to high energy electron $(>1 \mathrm{MeV})$, the low energy electron irradiation (LEEI, $<500 \mathrm{keV}$ ), does not generate $\mathrm{x}$ ray radiation and thus, does not require complex shielding [18]. LEEI works efficiently in inactivation of different viruses such as (influenza A (H3N8) and porcine reproductive and respiratory syndrome virus (PRRSV) [18]. On the other hand, ultraviolet radiation demonstrated an efficient role in inactivation of the enveloped MHV coronavirus [19], the enteric human adenovirus (HAdV) reported to be the most UVresistant virus [20]. Evaluation of the virus, radiation, temperatures are crucial factors [21]. The radiation dose used for viral inactivation should be calculated based on the virus concentration, the size, and the temperature of irradiation [22]. Therefore, it is important to assess the use of radiated inactivation and attenuation in virology. On the other hand, direct prediction of virus mutation 
amounts are significant to understand the evolution of the viruses and to struggle with them [23]. It is well known that radiation can cause mutations in bacteria and viruses. Therefore, charachterization of the radiation resistance and interaction properties of viruses provides the opportunity in terms of of risk assessment and future aspects. However, the literature review showed that no study was performed to investigate the radiation interaction mechanisms of this three types viruses viruses (SARS-CoV-CAS Number 587886-51-9, Influenza-CAS Number 141368-69-6 and SARS-CoV-2 GlycoProtein 6VSB.), which occured in an extraordinary way and creates serious concerns. This has strongly encouraged us to perform this extensive investigation. Taking all these current information and the extraordinary situation in the world into consideration, in this study, spike of 3 types of viruses (SARS-CoV-CAS Number 587886-51-9, Influenza-CAS Number 141368-69-6 and SARS-CoV-2 GlycoProtein 6VSB.) were modeled with the Monte Carlo simulation method. The vital radiation attenuation properties such as linear attenuation coefficients, energy absoption buildup factors (EABF), exposure buildup factors (EBF), relative dose distributions (RDD) were examined using advanced simulation methods. Moreover, the spike protein of SARS-CoV-2 is modelled from the structures in the Protein Data Bank [24]. As it can be seen from the Fig.1, spike protein part is the most external part of viruses. Therefore, it is highly expected to observe the first interactions in spike protein during the interaction between incoming radiation and virus structure. This study aimed to compare gamma ray attenuation properties of GlycoProtein structure of aforementioned virus types. It is well-known that radiation investigations such as dosimetry and radiation detection in micro-scale are difficult or physically impossible. Therefore, advanced simulation techniques and mathematical methods are highly required. Therefore, GlycoProtein structure of viruses sturcture has been modeled in MCNPX (version 2.6.0) Monte Carlo code [25]. We shall discuss simulation details and calculation steps in 
next sections. The hypohesis of this study is to observe a straight connection between radiation attenuation features and elemental properties of virues structure. In addition, this study aimed to create a new relationship hypothesis between mutation resistance of viruses and radiation attenuation properties for future investigations. In addition, the obtained outcomes from this theoretical study can provide a general idea on radiation attenuation properties of investigated virusues. Moreover, the outcome can also be useful for further investigations on radiation-virus interactions, efforts to improve the harmful effects of viruses.

\section{Materials and Methods}

\subsection{Viruses}

Coronaviruses have five structural proteins in their structures: the Spike (S), Membrane (M), Envelope (E) glycoproteins, Hemagglutinin Esterase (HE) and Nucleocapsid (N) protein (See Figure 1). All viruses have envelope proteins and $\mathrm{N}$ protein in their structure but HE is found only in some beta coronaviruses such as SARS-CoV-2 virus. The spike protein of SARS-CoV-2 is modelled from the structures in the Protein Data Bank [24]. The cartoon depiction of the protein is given in Fig. 2 from different viewpoints. (PDB ID: 6VSB) [26] . Mass weight percantage of SARS-CoV-2 is calculated with Materials Studio program and given in Table 1. These weight percentage of atoms are approximately similar for big size proteins. For example for Influenza spike protein weight percantage of $\mathrm{C}$ atom is $51.53 \%, \mathrm{H}$ atom $9.39 \%, \mathrm{O}$ atom $22.11 \%, \mathrm{~N}$ atom 16.10, S atom $0.88 \%$. Since viruses are composed of complex proteins, lipid layer and nucleic acids, the their buoyant densities are obtained by a combination of sucrose and cesium chloride density gradient equilibrium centrifugation techniques and proteins have approximately $1.3 \mathrm{~g} / \mathrm{ml}$ (with Sedimentation coefficients between 1-10) (Fig. 2), nucleic acids have $1.7 \mathrm{~g} / \mathrm{ml}$ and overall 
viruses have 1.35-1.4 g/ml bouyant densities (See Fig.3) [27]. In this study spike protein structure and its atom weight percentages and $1.3 \mathrm{~g} / \mathrm{ml}$ bouyant density are used in gamma radiation Monte Carlo simulations.

\subsection{Monte Carlo simulations}

In this study, version 2.6.0 of MCNPX [25] (Monte Carlo N-Particle eXtended) general-purpose Monte Carlo code was utilized for extensive investigation of mass attenuation coefficients of three different types of viruses namely SARS-CoV-CAS Number 587886-51-9, Influenza-CAS Number 141368-69-6 and SARS-CoV-2 GlycoProtein 6VSB considering their GlycoProtein part. A virus structure has been modeled considering essential parts. The GlycoProtein part cell envirionment of viruses has been filled with $M 1$ definition, which has been defined in the part of material definition in MCNPX input file. Next, $M 1$ has been defined considering the elemental mass fractions of SARS-CoV-CAS Number 587886-51-9, Influenza-CAS Number 141368-69-6 and SARS-CoV-2 GlycoProtein 6VSB, respectively. The Lambert-Beer law was employed for determination of mass attenuation coefficients (see Fig $\mathbf{4 b}$ ). On the other hand, the mass attenuation coefficients were defined for each photon energies at the range of $0.015-15 \mathrm{MeV}$. This process (cell definition and mass attenuation coefficients calculations) has been repeated for each virus structures. The appearance of modeled simulation setup can be seen in Figure 4a. It is worth to be mention that visualization of modeling simulation setup was performed in Visual Editor of $\operatorname{MCNPX}(\mathrm{VE}$ X_22S).

\subsection{Radiation attenuation properties}

In this study, Lambert-Beer's law was used with the help of the following equation to calculate the mass attenuation coefficients $\left(\mu_{\rho}\right)$ of spine protein structure of investigated viruses [28]. 
$I=I_{0} e^{-\mu_{\rho} t}$

In the formula, $\rho$ denotes the density, $t$ denotes the material thickness and $\mu_{\rho}$ denotes the mass attenuation coefficient. Units are $\mathrm{g} / \mathrm{cm}^{3}, \mathrm{~cm}$ and $\mathrm{cm}^{2} / \mathrm{g}$, respectively. Considering the mixture of elements, the mass attenuation coefficient is calculated by the equation [29] (2).

$$
\frac{\mu}{\rho}=\mu_{\rho}=\frac{N_{A}}{M} \sigma_{t o t}=\sum_{i} w_{i}(\mu / \rho)_{i}
$$

wheree, $\mathrm{w}_{\mathrm{i}}$ is a wieght fraction of $\mathrm{i}^{\text {th }}$ element in the virus structure. With the help of the calculation of the mass attenuation coefficient, which is the key parameter to calculate the attenuation parameters, calculations of MFP values were also calculated (Equation 3). The Mean Free Path (MFP) can be described for both charged particles and photons. It represents the path that a gamma photon takes without interacting with the material and is expressed in terms of linear attenuation coefficient [30].

$$
\mathrm{MFP}=1 / \mu
$$

The term of build-up factor can be defined as the ratio of the amount of radiation at a given location to the amount of radiation coming from this location without interference. The agglomeration factor is defined as the function of the depth of interaction, ie the mean free path. The mean free path (MFP) varies with linear distance, the unit mean free path depends on the absorption coefficient of the material medium and corresponds to a linear value. Exposure buildup factors $(\mathrm{EBF})$, Energy absorption buildup factors $(\mathrm{EABF})$ are the determining quantities in radiation attenuation and radiation measurement studies. In the interaction of gamma radiation with the target material, two radiation components are formed inside or beyond the attenuator; collided and un-collided photons. Compton partial attenuation coefficient $\left(\mu_{\rho}\right)_{\text {Compton }}$ and total attenuation coefficient $\left(\mu_{\rho}\right)$ Total values were obtained for three types of viruses in the energy range of $0.015-$ 
15.0 MeV to calculate the EBF and EABF buildup factors. Then the equivalent atomic number $\left(Z_{\mathrm{eq}}\right)$ values of the glass samples were calculated by matching the $\left(\mu_{\rho}\right)_{\text {Compton }} /\left(\mu_{\rho}\right)_{\text {Total }}$ ratio of the particular energy to the corresponding ratios of the elements of the same energy. When the $\left(\mu_{\rho}\right)_{\text {Compton }} /\left(\mu_{\rho}\right)_{\text {Total }}$ ratio is between two consecutive rates, its interpolation is calculated by the following equation:

$Z_{e q}=\frac{Z_{1}\left(\log R_{2}-\log R\right)+Z_{2}\left(\log R-\log R_{1}\right)}{\log R_{2}-\log R_{1}}$

where $\mathrm{Z} 1$ and $\mathrm{Z} 2$ are the atomic numbers of the elements corresponding to the ratios of $\mathrm{R} 1$ and $\mathrm{R} 2$, respectively. $\mathrm{R}$ indicates the proportion of glasses studied in a particular energy.

The EBF and EABF values of Hematite doped glasses were estimated for gamma energies up to $15 \mathrm{MeV}$ and for penetration depth up to $40 \mathrm{mfp}$ by using G-P method [31]:

$$
\begin{array}{cc}
C=\frac{C_{1}\left(\log Z_{2}-\log Z_{e q}\right)+C_{2}\left(\log Z_{e q}-\log Z_{1}\right)}{\log Z_{2}-\log Z_{1}} & \\
B(E, X)=1+\left(\frac{b-1}{K-1}\right)\left(K^{x}-1\right) & \text { for } K \neq 1 \\
B(E, X)=1+(b-1) x & \text { for } K=1
\end{array}
$$

where,

$$
K(E, x)=c x^{a}+d \frac{\tanh \left(\frac{x}{X_{K}}-2\right)-\tanh (-2)}{1-\tanh (-2)} \quad \text { for } x \leq 40 \mathrm{mfp}
$$

Moreiver, the G-P fitting parameters (a,b,c,d and Xk) were utilized. These equations contain $\mathrm{x}$ as the distance between the source and detector. At $1 \mathrm{mfp}$, the $\mathrm{EBF}$ is coded by $\mathrm{b} . \mathrm{K}(\mathrm{E}, \mathrm{X})$ factor has a meaning of dose multiplication.

Finally, Relative Dose Distribution (RDD) values were determined for investgated viruses. The term of RDD at distance $\mathrm{X}$ is calculated using the formula below [32]: 


$$
R D D=\frac{\exp (-\mu \mathrm{x})(\mathrm{EBF})}{x^{2}}
$$

\section{Results and discussions}

For the three different types of viruses SARS-CoV-CAS Number 587886-51-9, Influenza-CAS Number 141368-69-6 and SARS-CoV-2 GlycoProtein 6VSB), chemical structures along with densities and weight fractions of GlycoProteins are reported in Table 1. Linear attenuation coefficients (LAC) values are computed at the energy range between 0.015 to $15 \mathrm{MeV}$ by using MCNPX code. The results are shown in Fig.5 . From the figure, it can be seen that LAC values are corelated to the applied energy as well as the chemical composition of the GlycoProteins. While the applied energy increases up to $15 \mathrm{MeV}$, the LAC values shows a declining rate. Among the investigated viruses, linear attenuation coefficients values of (CAS Number 587886-51-9) were higher than Influzenza and Covid-19. To evaluate attenuation characteristic and quality, linear absorption coefficient (LAC) values as a function of photon energy were determined (See Fig.6). The results showed that attenuation properties of each type of GlycoProtein structure were higher in low energy region. However, similar behaviors with LAC trend was observed in absorption characteristic of investigated viruses. The term of mean free path (MFP) is important parameter to determine the average distance of radiation in material environment. The MFP values for the studied viruses are reported in the Fig. 7. The values of the energy absorption buildup factor $(\mathrm{EABF})$ and the exposure buildup factor $(\mathrm{EBF})$ prove useful when choosing a replacement composite material instead of an element for a specific energy region. As such EABF and EBF are key to define the absorption of the material. In this study, EBF-EABF values are calculated by using G-P fitting approach, which a common method used by various researcher. The penetration depth for the calculation is at 1-5-10-20-40 MFP respectively. The Fig.8 and Fig.9 list the EBFEABF values in the energy levels up to $15 \mathrm{MeV}$ for Sars-Cov, Influenza and Sars-Cov-2. From 
the figure, it can be seen that different energies yield various incidents. Photoelectric effect, Compton scattering, and pair production are the three interactions taking place between energy and matter. Photoelectric effect happens as electrons are released by matter while subjected to electromagnetic radiation. Photoelectric effects occurs at low energy regions yielding increasing rate of $\mathrm{EBF}$ and $\mathrm{EABF}$ with an inverse relations to $\mathrm{E}^{3.5}$. EBF-EABF values decline with the increasing energy after reaching its maximum at $0.1 \mathrm{MeV}$. Compton scattering is the result of a photon of high energy occuring in the the intermediate energy region, it yields higher EBF-EABF values owing to multiple scattering processes. Pair production is a simple translation of the radiant energy into matter. By this way the gamma-ray is converted into matter in the shape of a pair of electrons (negatron and positron) charged negatively and positively. Pair production occurs just for high-energy gamma-rays yielding lower EAB-EABF. Sars-Cov is the smallest possible EBFEABF element among the studied sample with a maximum value of Zeq. Fig.s 7 and 8 shows that since the higher energy penetrations move through the substance, higher EBF-EABF values can be seen in the energy from $0.015 \mathrm{Mev}$ to $15 \mathrm{MeV}$ and up to $40 \mathrm{mfp}$. It is known that chemical composition has significant effects on the EBF and EABF. As it can be seen in the Fig.7-8, SarsCov, Sars-Cov-2 possess the smallest EBF-EABF values at the low energies. EABF and EBF values were found to increase with declining Zeq of all of the investigated samples. This inverse relation between Zeq and EBF-EABF is recorded in the Table 2, 3 and 4. Having the minimum $\mathrm{Z}_{\mathrm{eq}}$ Influenza possess the largest EBF-EABF, while Sars-Cov yields the maksimum $\mathrm{Z}_{\mathrm{eq}}$ and smallest EBF-EABF. A high value of Zeq for a material means it's a strong absorber of radiation. Energy matter interaction can be divided into three areas for Zeq values as well. In the high and intermediate energies, $Z_{\text {eq }}$ 's dependence on energy declines and maxsimum $Z_{\text {eq }}$ is found in intermediate energy regions owing to the dominating Compton scattering process. Vis-à-vis 
chnaging energies, the variation of EBF and EABF values for the the Sars-Cov, Influenza and Sars-Cov-2 samples are reported in Figs. 8 and 9. Thanks to the K absorption edge of Sulfur an abrupt peak is located in this region in the low region between $0.015-0.1 \mathrm{MeV}$ and decrease swiftly after 0.1 MeV. Here, EBF-EABF values get to the minimum levels for all investigated viruses. In the 0.015-0.1 MeV, EBF and EABF incrementally rise and sharply decline after $0.1 \mathrm{MeV}$. EBFEABF values show an increasing trend yet again for high penetration depths (15-40 Mfp). From Figs. 8 and 9, the EBF and EABF values are lowered by growing the percentage of sulfur in the virus samples and hence the total EABF and EBF values were obtained by the Sars-Cov. The smaller EBF-EABF implies improved properties of radiation shielding. So, we may assume that Sars-Cov has greater value than Sars-Cov-2 in terms of the radiation shielding. Sars-Cov, with the maximum sample of Zeq virus among the investigated virus samples, arrived at the smallest EABF-EBF as the SARS-Cov-2, with minimum sample of Zeq, reaches the largest EBF-EABF. A technique for the quantitative evaluation of the relative dose distribution (RDD) is a measurement of the distance $\mathrm{x}$ to the EBF values. Variations of the RDD were found as a feature of distance (x) for various samples of viruses. Figure $\mathbf{1 0}$ indicates the variation in the relative dose distribution of Sars-Cov, Influenza, and Sars-Cov-2 viruses at penetration depths of 10, 20, 30 and $40 \mathrm{mfp}$ for specific event energies up to $15 \mathrm{MeV}$ and for various distances up to $1 \mathrm{~cm}$. As seen in these statistics, the RDD values increase to maturity based on the samples 'penetration depth and chemical composition. Of both tests, the RDD values grow quickly as the photon energy rises to 0.1 MeV. Increases in photon energy often contribute to decrease of the RDD values. Moreoever, Figure 11 shows that, RDD behaviors of investigated viruses did not changed in different penetration depths (10 mfp, $20 \mathrm{mfp}, 30 \mathrm{mfp}, 40 \mathrm{mfp})$. However, slight differences in the lower penetration depths were relatively increased in higher penetration depths. 


\section{Conclusion and future aspects}

Although many studies have been carried out about the radiation and biological interaction mechanism in the historical process, some issues still remain mysterious. One of these issues is radiation and virus interactions. Different types of studies have been performed in terms of inactivation of viruses using ionizing and non ionizing radiations. On the other hand, many applications are under investigation. It is well-known that radiation investigations such as dosimetry and radiation detection in micro-scale are difficult or physically impossible. Therefore, advanced simulation techniques and mathematical methods are highly required. The importance of investigated virus types and this difficult situation in the world strongly encouraged us to do this extensive theoretical study and to examine the radiation resistance properties of these viruses. Of course, outcomes of this investigation should be considered as a first step. Therefore, experimental and clinical investigations will be planned, immediately. On the other hand, obtained results would be useful to compare the mutation posibilites of Covid-19 with SARS-CoV and Influenza in terms of ionizing radiation resistance. As a result of the study, we could say that the most resistant was observed in SARS-CoV when compared with Influenza and Covid-19. It could be one of the reasons for SARS-CoV's resistance to mutation from its outbreak time. On the other hand, Covid-19 is more resistant to radiation than Influenza. Therefore it could be expected that Covid-19 would have the mutating ability as Influnza has. Furthermore, it is not predictable if Covid-19 will resist to sunlight's UV radiation. Because a large number of people are expecting that its spread will decline when the weather gets warms up due to sunlight. To clarify this problem, some experiments should be carried out in the lab conditions. The obtained outcomes from this theoretical study has provided a general idea on radiation attenuation properties of investigated virusues. The hypothesis of recent investigation was to observe a direct behavior relationship 
between spike protein and incoming radiation. The obtained result was approved our hypothesis and slight differences were observed. Of course, this hypothesis should be approved by experimental and clinical investigation in near future.

\section{Declaration of Interests}

All authors declare no competing interest

\section{Acknowledgment}

The authors would like to acknowledge the Johns Hopkins University for open sourcing their dataset. Also, like to acknowledge the World Health Organization (WHO) for their timely situational reports on 2019-nCoV.

\section{$\underline{\text { References }}$}

[1] Dey, S. K., Rahman, M. M., Siddiqi, U. R., \& Howlader, A. (2020). Analyzing the Epidemiological Outbreak of COVID-19: A Visual Exploratory Data Analysis (EDA) Approach. Journal of Medical Virology.

[2] Ibrahim, I. M., Abdelmalek, D. H., Elshahat, M. E., \& Elfiky, A. A. (2020). COVID-19 Spike-host cell receptor GRP78 binding site prediction. Journal of Infection.

[3] Andersen, K. G., Rambaut, A., Lipkin, W. I., Holmes, E. C., \& Garry, R. F. (2020). The proximal origin of SARS-CoV-2. Nature Medicine, 1-3.

[4] Ghinai, I., McPherson, T. D., Hunter, J. C., Kirking, H. L., Christiansen, D., Joshi, K.,\& Fricchione, M.

J. (2020). First known person-to-person transmission of severe acute respiratory syndrome coronavirus 2 (SARS-CoV-2) in the USA. The Lancet, 1-8.

[5] Xu, Y., Li, X., Zhu, B., Liang, H., Fang, C., Gong, Y., \& Zhang, H. (2020). Characteristics of pediatric SARS-CoV-2 infection and potential evidence for persistent fecal viral shedding. Nature Medicine, 1-4. 
[6] Van Doremalen, N., Bushmaker, T., Morris, D. H., Holbrook, M. G., Gamble, A., Williamson, B. N., \& Lloyd-Smith, J. O. (2020). Aerosol and Surface Stability of SARS-CoV-2 as Compared with SARSCoV-1. New England Journal of Medicine

[7] Barker CA, Postow MA. Combinations of radiation therapy and immunotherapy for melanoma: a review of clinical outcomes. Int J Radiat Oncol Biol Phys 2014;88:986-997.

[8] McDonald JC. Industrial radiation processing: working behind the scenes. Radiat Prot Dosimetry 2004;109:173-174.

[9] Lowy, R. J., Vavrina, G. A., \& LaBarre, D. D. (2001). Comparison of gamma and neutron radiation inactivation of influenza A virus. Antiviral research, 52(3), 261-273.

[10] Block, S. S. (Ed.). (2001). Disinfection, sterilization, and preservation. Lippincott Williams \& Wilkins.

[11] Grassly NC. Immunogenicity and effectiveness of routine immunization with 1 or 2 doses of inactivated poliovirus vaccine: systematic review and meta-analysis. J Infect Dis 2014;210 Suppl 1:S439_ S446.

[12] Fulda, S., A.M. Gorman, O. Hori, A. Samali. 2010. Cellular stress responses: cell survival and cell death. International Journal of Cell Biology, 2010: 214074.

[13] Syaifudin, M., Nurhayati, S., Darlina, D., Lusiyanti, Y., \& Kisnanto, T. (2019). Irradiation of intraerythrocytic Plasmodium berghei with a fractionated dose of gamma rays does not effectively reduce the infectivity in mice Mus musculus. Aceh Journal of Animal Science, 4(1), 18-26.

[14] Lowy, R. J. (2005). Ionizing radiation inactivation of medically relevant viruses. Radiation Inactivation of Bioterrorism Agents, 365, 175.

[15] Gaidamakova, E.K.; Myles, I.A.; McDaniel, D.P.; Fowler, C.J.; Valdez, P.A.; Naik, S.; Maheshwari, R.K. Preserving immunogenicity of lethally irradiated viral and bacterial vaccine epitopes using a radioprotective Mn(2+)-peptide complex from Deinococcus. Cell Host Microbe 2012, 12, 117-124

[16] International Atomic Energy Agency. The Manual on Radiation Sterilization of Medical and Biological Material: The technical reports series. Vienna: 1973. pp. 62-63.

[17] Astill, J., Alkie, T., Yitbarek, A., Taha-Abdelaziz, K., Bavananthasivam, J., Nagy, É., ... \& Sharif, S. (2018). Examination of the effects of virus inactivation methods on the induction of antibody-and cellmediated immune responses against whole inactivated H9N2 avian influenza virus vaccines in chickens. Vaccine, 36(27), 3908-3916.

[18] Fertey, J., Bayer, L., Grunwald, T., Pohl, A., Beckmann, J., Gotzmann, G., ... \& Thoma, M. (2016). Pathogens inactivated by low-energy-electron irradiation maintain antigenic properties and induce protective immune responses. Viruses, 8(11), 319. 
[19] Walker, C. M., \& Ko, G. (2007). Effect of ultraviolet germicidal irradiation on viral aerosols. Environmental science \& technology, 41(15), 5460-5465.

[20] Pimenta, A. I., Guerreiro, D., Madureira, J., Margaça, F. M., \& Verde, S. C. (2016). Tracking Human Adenovirus Inactivation by Gamma Radiation under Different Environmental Conditions. Appl. Environ. Microbiol., 82(17), 5166-5173.

[21] House, C., House, J. A., \& Yedloutschnig, R. J. (1990). Inactivation of viral agents in bovine serum by gamma irradiation. Canadian journal of microbiology, 36(10), 737-740.

[22] Salehi, B., Motamedi-Sedeh, F., Madadgar, O., Khalili, I., Ghalyan Chi Langroudi, A., Unger, H., \& Wijewardana, V. (2018). Analysis of antigen conservation and inactivation of gamma-irradiated avian influenza virus subtype H9N2. Acta microbiologica et immunologica Hungarica, 65(2), 163-171.

[23] Rafael Sanjuán, Miguel R. Nebot, Nicola Chirico, Louis M. Mansky, Robert Belshaw Journal of Virology Sep 2010, 84 (19) 9733-9748; DOI: 10.1128/JVI.00694-10

[24] H.M. Berman, J. Westbrook, Z. Feng, G. Gilliland, T.N. Bhat, H. Weissig, I.N. Shindyalov, P.E. Bourne. (2000) The Protein Data Bank Nucleic Acids Research, 28: 235-242.

[25] RSICC Computer Code Collection, MCNPX User's Manual Version 2.4.0. Monte Carlo N-Particle Transport Code System for Multiple and High Energy Applications, (2002).

[26] Wrapp, D.; Wang, N.; Corbett, K. S.; Goldsmith, J. A.; Hsieh, C.-L.; Abiona, O.; Graham, B. S.; McLellan, J. S., Cryo-EM structure of the 2019-nCoV spike in the prefusion conformation. Science 2020, 367 (6483), 1260.

[27] Mazzone, Horace M. CRC Handbook of Viruses: Mass-Molecular Weight Values and Related Properties. Boca Raton, FL: CRC Press, 1998.

[28] H.O. Tekin, Mesut Karahan, Turker Tekin Erguzel, Tugba Manici, Muhsin Konuk. Radiation Shielding Parameters of Some Antioxidants using Monte Carlo Simulation. Journal of Biological Physics, 44(4), 579-590. https://doi.org/10.1007/s10867-018-9507-6

[29] M. A. M. Uosif, A.M.A. Mostafa, Shams A.M. Issa, H.O. Tekin, Z.A. Alrowaili, O. Kilicoglu. Structural, mechanical and radiation shielding properties of newly developed tungstenlithium borate glasses: An experimental study. Journal of Non-Crystalline Solids. Journal of Non-Crystalline Solids 532 (2020) 119882. https://doi.org/10.1016/j.jnoncrysol.2019.119882

[30] Yasser B. Saddeek, Shams A.M. Issa, Thamer Shalih Al-Harbi, H.O. Tekin, O. Kilicoglu, T.T. Erguzel, Kamal A. Aly, Mahmoud Ahmad. Improvement of radiation shielding properties of some tellurovanadate based glasses. Physica Scripta. Accepted Manuscript Online: 4 November 2019. https://doi.org/10.1088/1402-4896/ab541d 
[31] H.O. Tekin, E. Kavaz, Athanasia Papachristodoulou, M.Kamislioglu, O.Agar, E.E.Altunsoy Guclu, O.Kilicoglu, M.I. Sayyed. Characterization of $\mathrm{SiO} 2-\mathrm{PbO}-\mathrm{CdO}-\mathrm{Ga} 2 \mathrm{O} 3$ glasses for comprehensive nuclear shielding performance: Alpha, proton, gamma, neutron Radiation. Ceramics International 45 (2019) 19206 - 19222. https://doi.org/10.1016/j.ceramint.2019.06.168

[32] Shams A.M. Issa, G. Susoy, Atif Mossad Ali, H.O. Tekin, Y.B. Saddeek, Ali Al-Hajry, Hamed Algarni, P.S. Anjana, O. Agar. The effective role of La2O3 contribution on zinc borate glasses: radiation shielding and mechanical properties. Applied Physics A. (2019) 125:867. https://doi.org/10.1007/s00339019-3169-5 


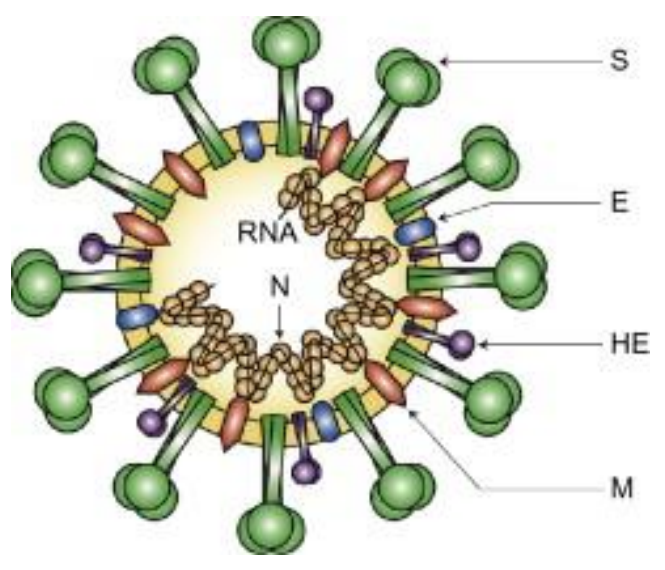

Figure 1: Coronavirus structure with structural proteins. N: Nucleocapsid protein; S: Spike protein, M: Membrane protein, HE: Hemagglutinin-Esterase and E: Envelope protein

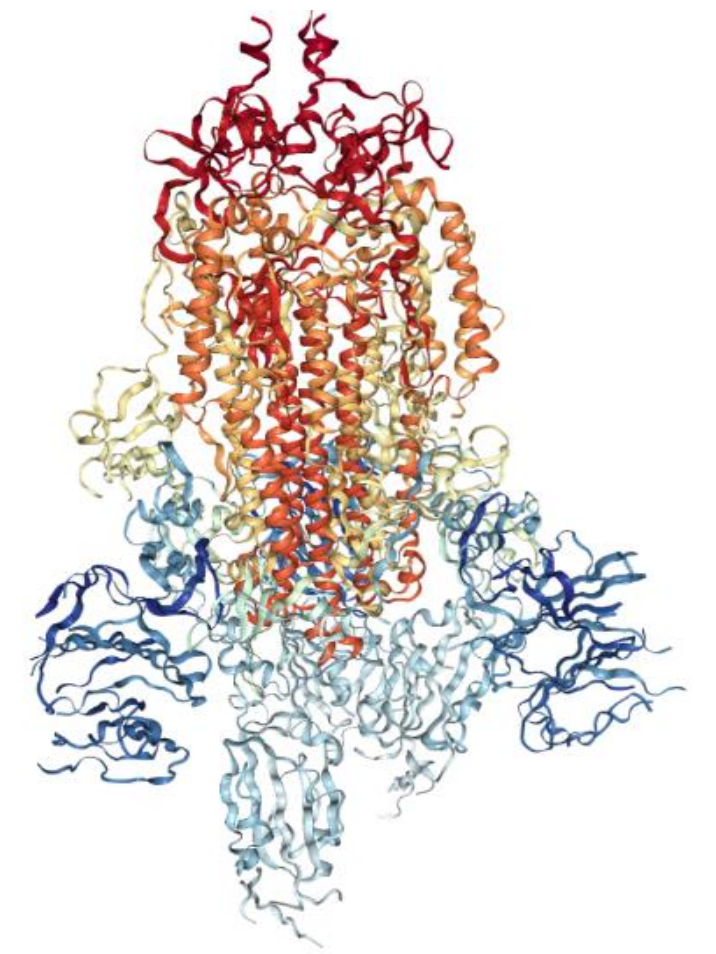

(a)

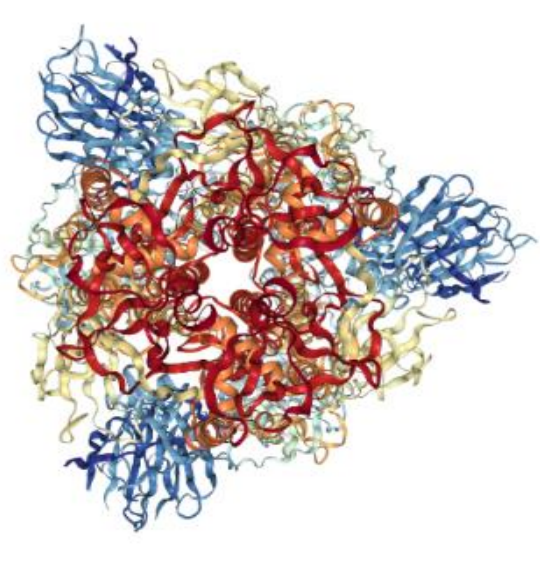

(b)

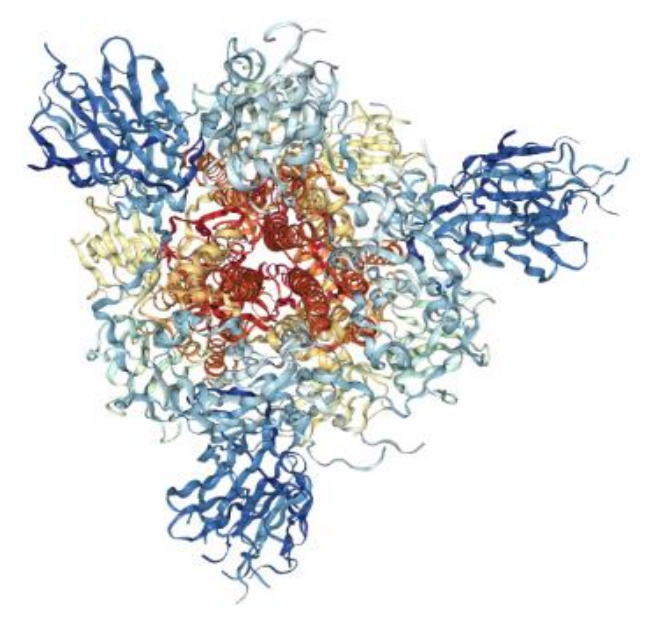

(c)

Figure 2. The Structure of Human Coronavirus GlycoProtein (6VSB) (a) side view (b) top view (c) bottom view 


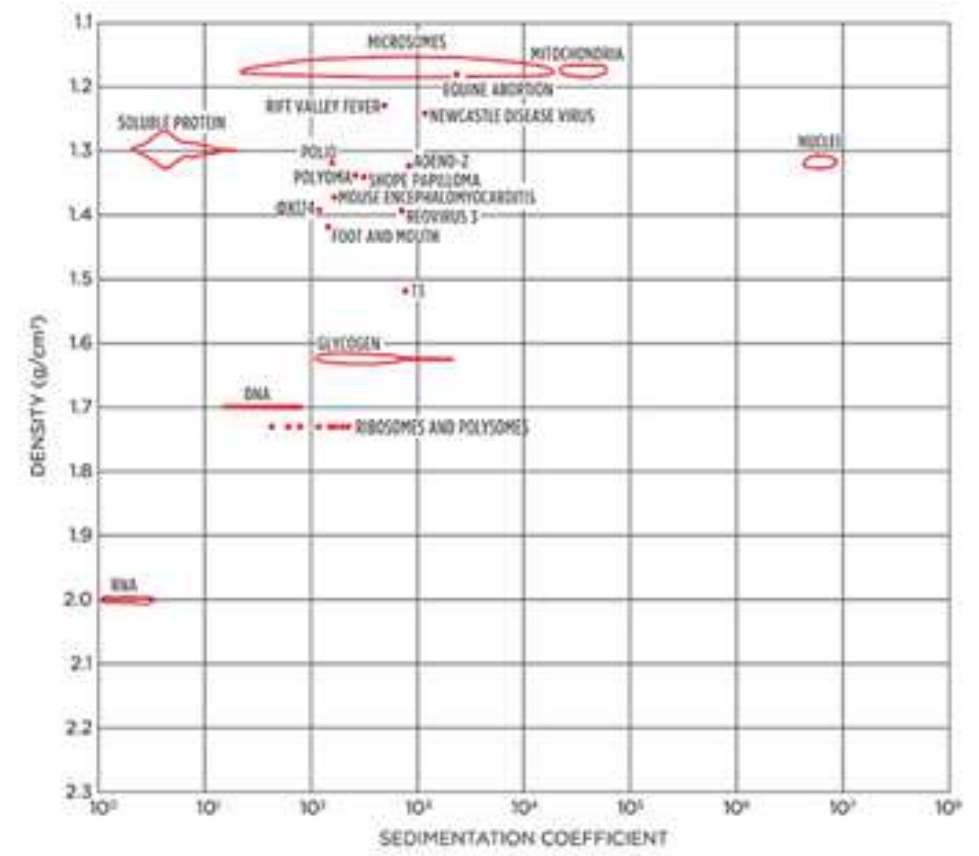

Figure 3. Virus/Cell Organelle densities and S values

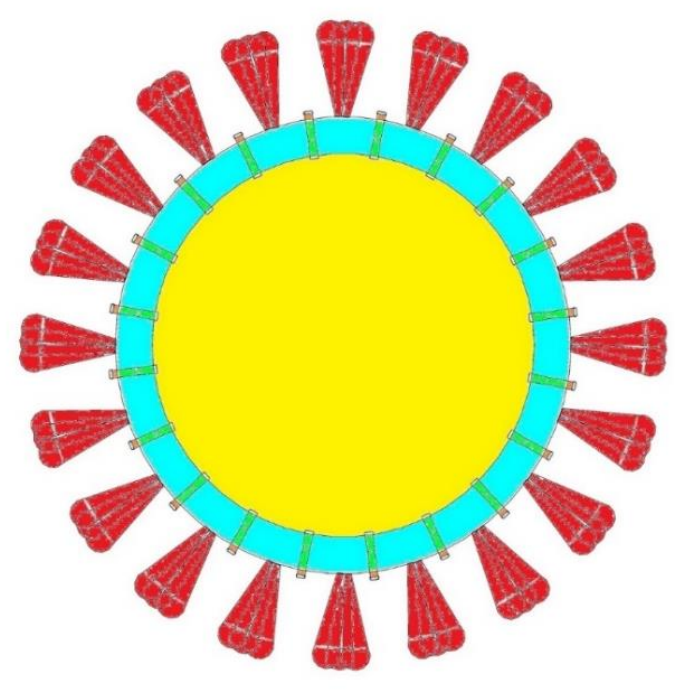

(a)

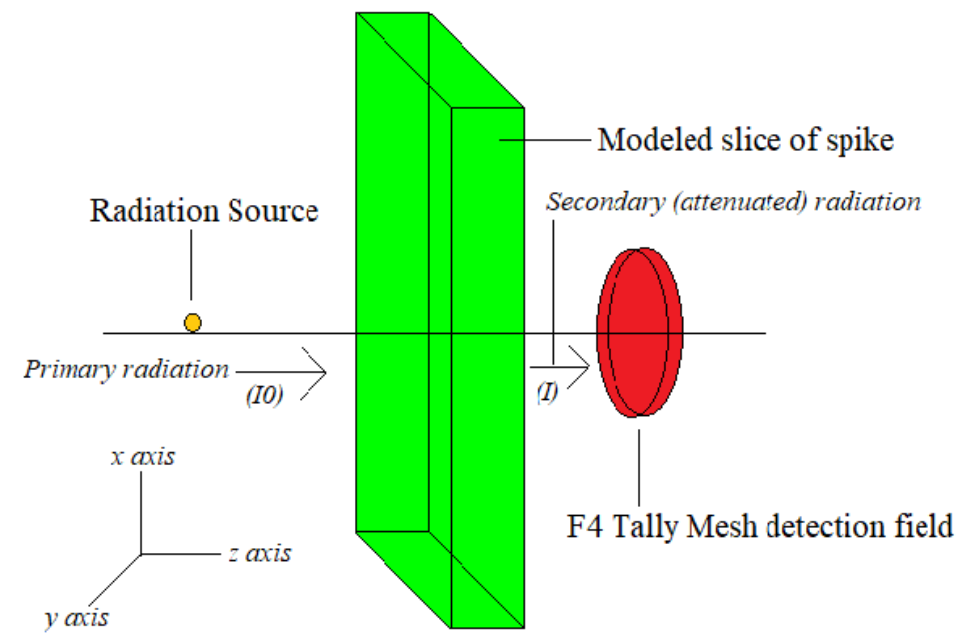

(b)

Figure 4. (a) Axial view of modeled virus structure in MCNPX code (b) Radiation transmission setup for calculation of attenuation coefficients 


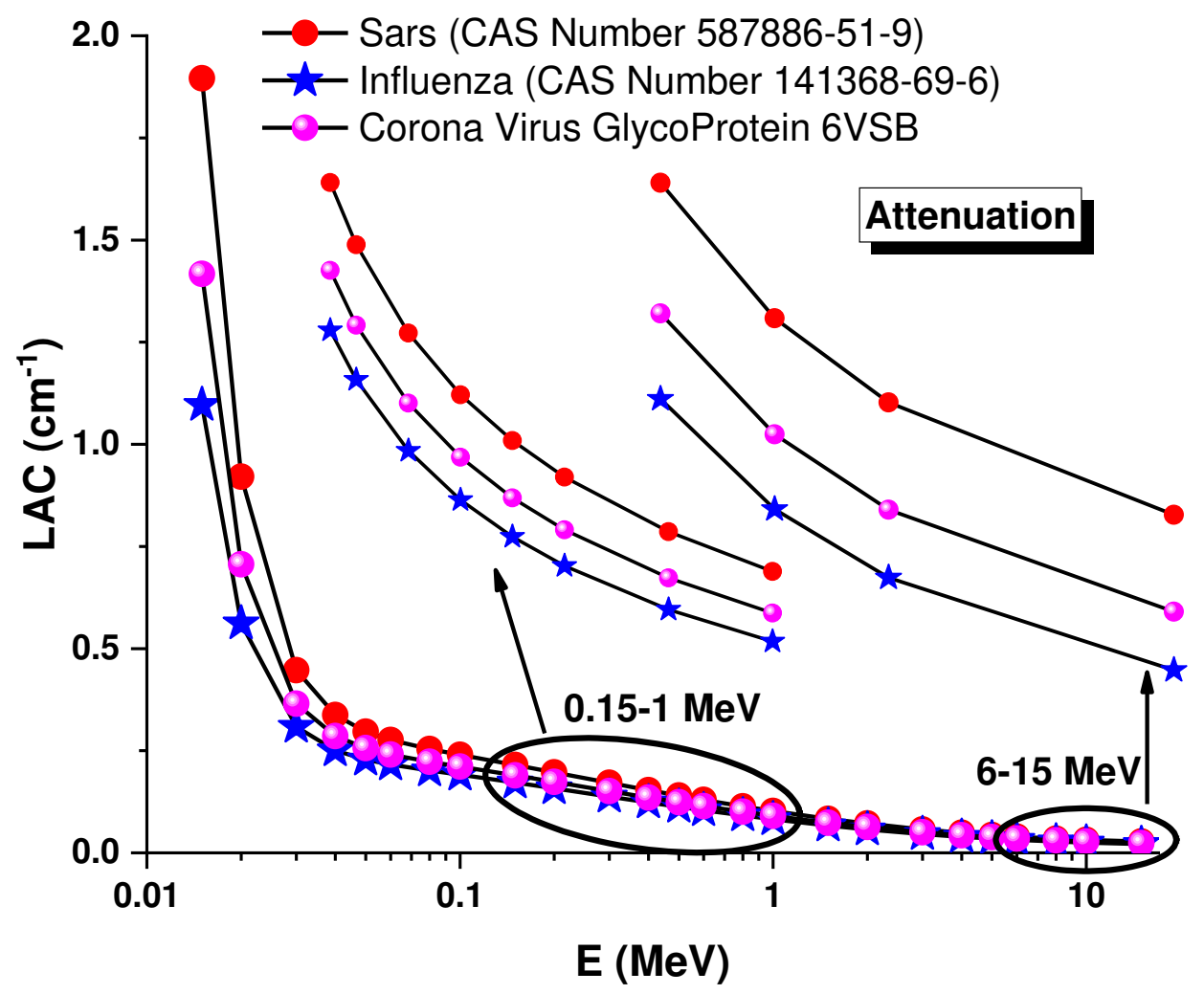

Fig. 5 Linear attenuation coefficient (LAC) values as a function of photon energy of Sars (CAS Number 587886-51-9), Influenza (CAS Number 141368-69-6) and Covid-19 GlycoProtein 6VSB.

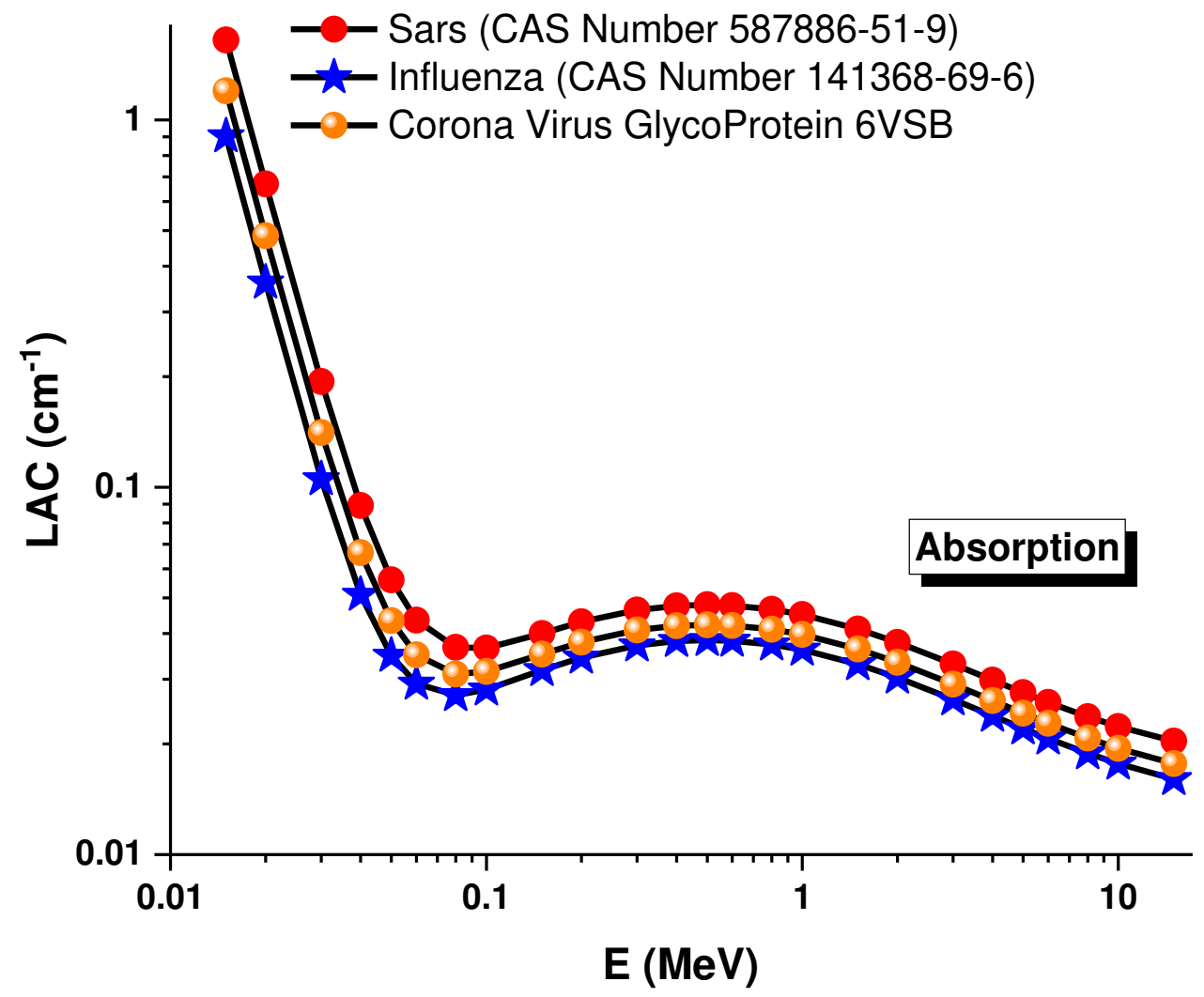

Fig. 6 linear absorption coefficient (LAC) values as a function of photon energy of Sars (CAS Number 58788651-9), Influenza (CAS Number 141368-69-6) and Covid-19 GlycoProtein 6VSB. 


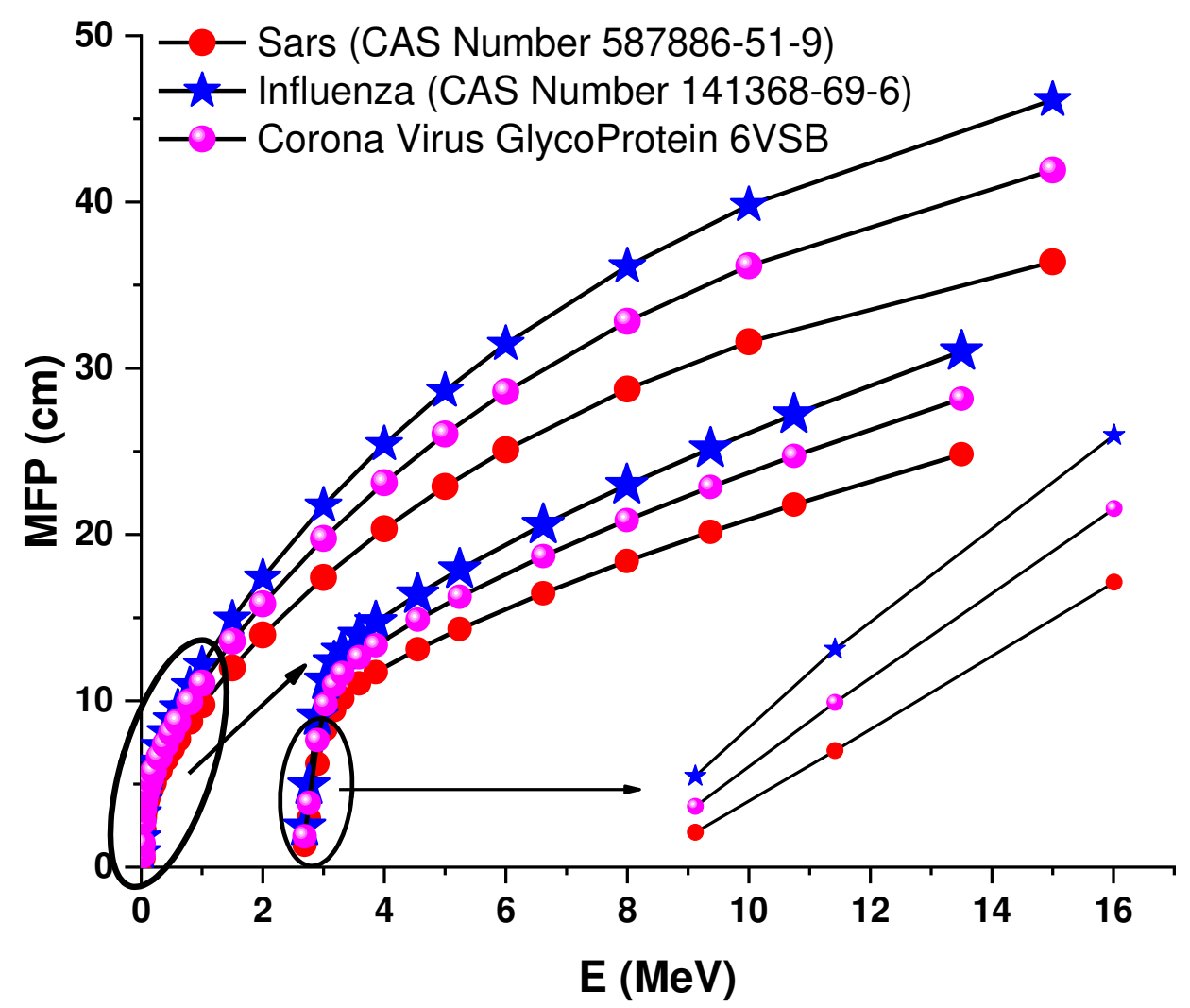

Fig. 7 Mean free path (MFP) values as a function of photon energy of Sars (CAS Number 587886-51-9), Influenza (CAS Number 141368-69-6) and Covid-19 GlycoProtein 6VSB.

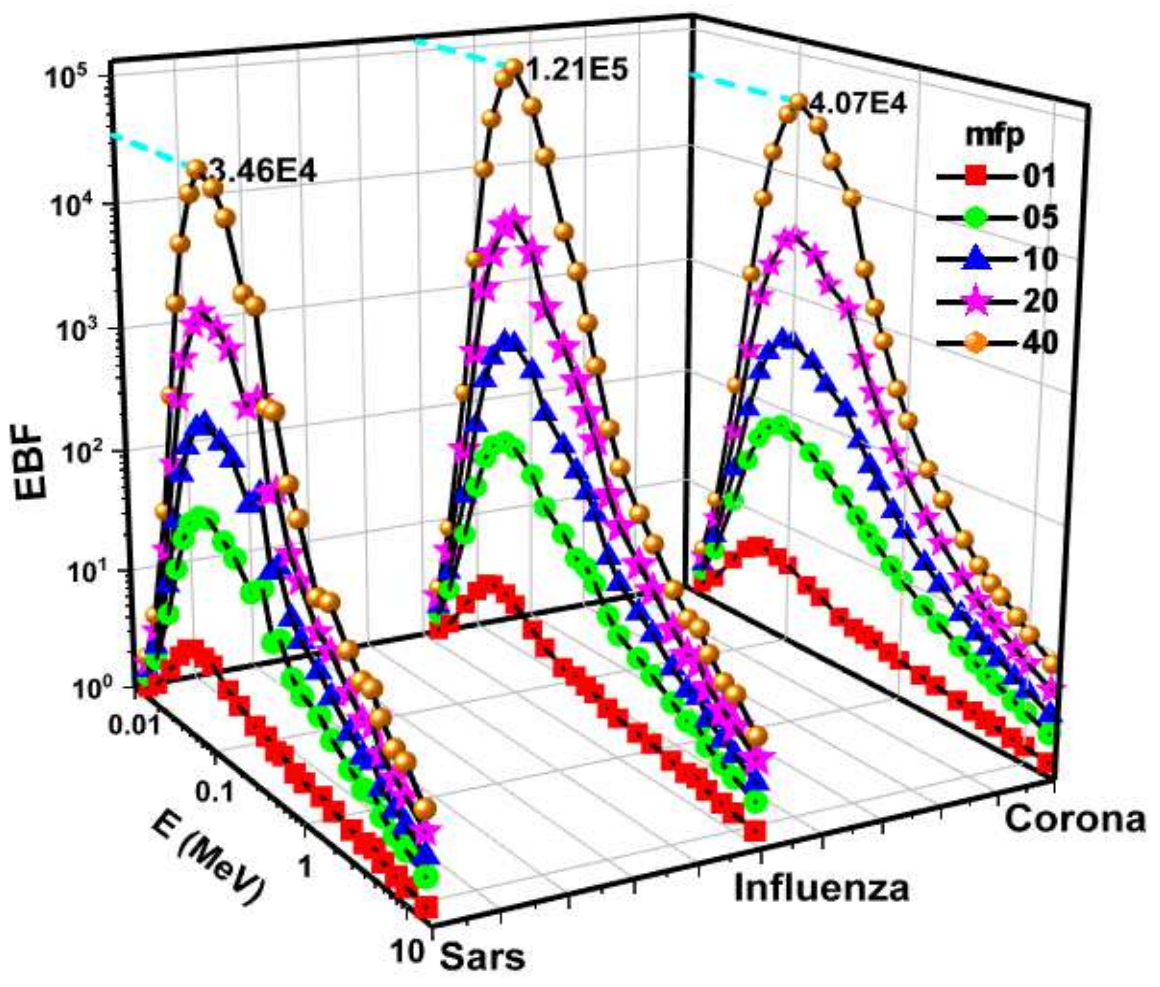

Fig. 8 Exposure buildup factor (EBF) values as a function of photon energy of Sars (CAS Number 587886-519), Influenza (CAS Number 141368-69-6) and Covid-19 GlycoProtein 6VSB. 


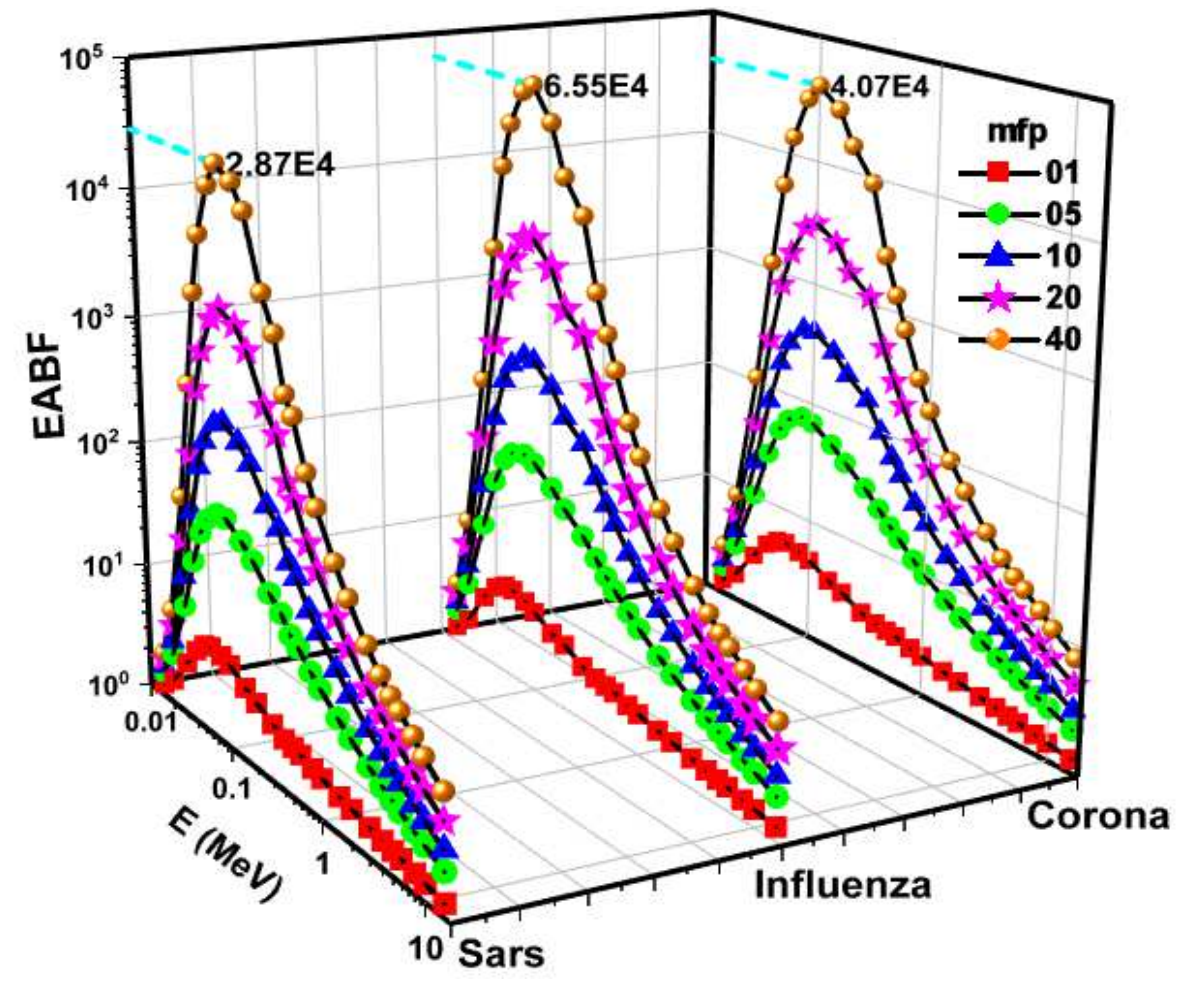

Fig. 9 Energy absorption buildup factor (EABF) values as a function of photon energy of Sars (CAS Number 587886-51-9), Influenza (CAS Number 141368-69-6) and Covid-19 GlycoProtein 6VSB.

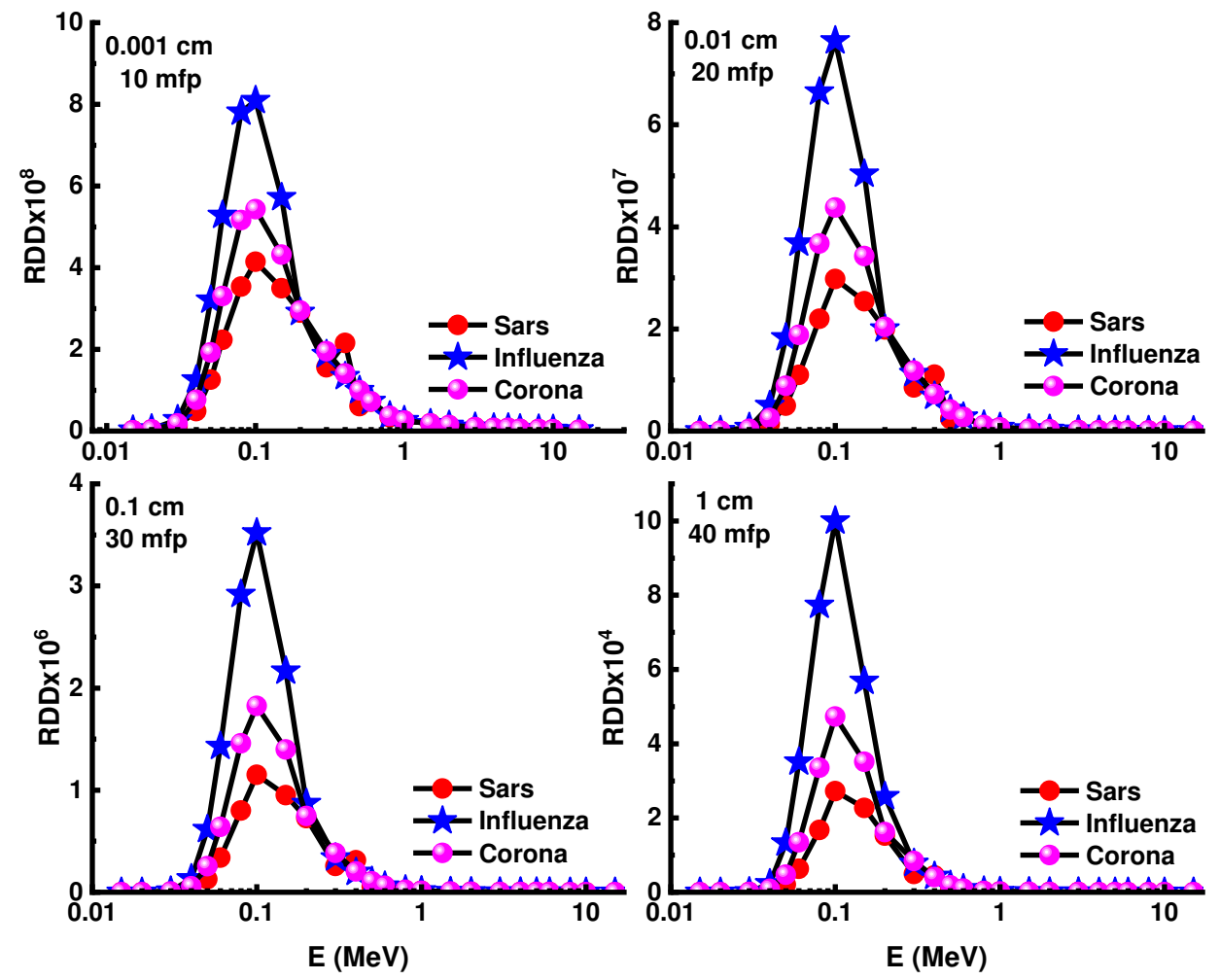

Fig. 10 Relative dose distribution (RDD) values as a function of photon energy at different penetration depth (mfp) and distances (cm) of Sars (CAS Number 587886-51-9), Influenza (CAS Number 141368-69-6) and Covid-19 GlycoProtein 6VSB. 

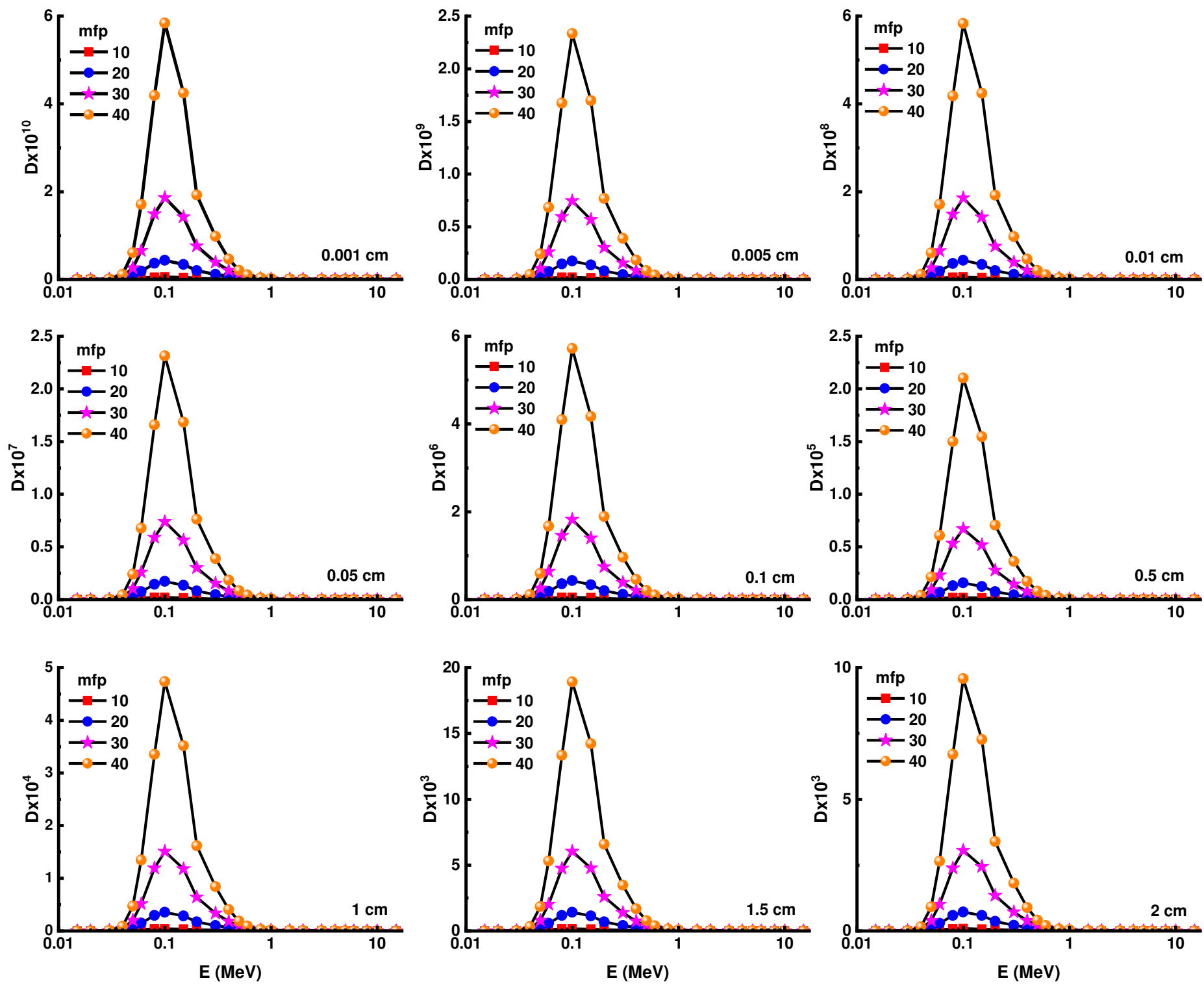

Fig. 11 Relative dose distribution values as a function of photon energy at different penetration depth (mfp) and distance $(\mathrm{cm})$ of Covid-19 GlycoProtein 6VSB. 
Table 1 . Elemental mass percentages (\%) of investigated virüs types

\begin{tabular}{|l|l|l|l|}
\hline Atom Type & Influenza & Sars & Covid-19 \\
\hline Carbon $(\mathrm{C})$ & 60.9129 & 49.8293 & 52.38 \\
\hline Hydrogen $(\mathrm{H})$ & 7.8242 & 7.5397 & 9.51 \\
\hline Nitrogen $(\mathrm{N})$ & 13.0474 & 18.4896 & 15.59 \\
\hline Oxygen $(\mathrm{O})$ & 18.2155 & 22.1258 & 21.55 \\
\hline Sulfur $(\mathrm{S})$ & 0 & 2.0156 & 0.97 \\
\hline
\end{tabular}


Table 2 (EBF and EABF) G-P fitting coefficients (b, c, a, $X_{k}$ and d) of Sars (CAS Number 587886-51-9).

\begin{tabular}{|c|c|c|c|c|c|c|c|c|c|c|c|}
\hline \multirow{2}{*}{$\begin{array}{c}\mathbf{E} \\
(\mathrm{MeV})\end{array}$} & \multirow[t]{2}{*}{$\mathbf{Z}_{\mathrm{eq}}$} & \multicolumn{5}{|c|}{ EBF } & \multicolumn{5}{|c|}{ EABF } \\
\hline & & $\mathbf{b}$ & c & $\bar{a}$ & $\overline{\mathbf{X}_{\mathbf{k}}}$ & d & $\mathbf{b}$ & $\bar{c}$ & $\overline{\mathbf{a}}$ & $\overline{\mathbf{X}_{\mathrm{k}}}$ & $\bar{d}$ \\
\hline 0.015 & 7.04 & 1.2302 & 0.4730 & 0.1718 & 14.2803 & -0.0863 & 1.2322 & 0.4822 & 0.1646 & 14.5034 & -0.0796 \\
\hline 0.020 & 7.10 & 1.5239 & 0.5778 & 0.1332 & 14.9645 & -0.0652 & 1.5389 & 0.5703 & 0.1370 & 14.8564 & -0.0680 \\
\hline 0.030 & 7.16 & 2.5275 & 0.8216 & 0.0584 & 15.8188 & -0.0408 & 2.6231 & 0.8123 & 0.0626 & 15.3063 & -0.0389 \\
\hline 0.040 & 7.19 & 3.7854 & 1.2219 & -0.0402 & 13.5543 & 0.0127 & 3.8329 & 1.2163 & -0.0386 & 13.6874 & 0.0110 \\
\hline 0.050 & 7.20 & 4.8315 & 1.5688 & -0.1009 & 13.7841 & 0.0424 & 4.7397 & 1.5626 & -0.0997 & 13.8431 & 0.0416 \\
\hline 0.060 & 7.22 & 5.2973 & 1.8484 & -0.1412 & 13.6965 & 0.0643 & 5.0247 & 1.8382 & -0.1397 & 13.7464 & 0.0629 \\
\hline 0.080 & 7.29 & 5.2377 & 2.1644 & -0.1796 & 13.4288 & 0.0817 & 4.9220 & 2.1313 & -0.1752 & 13.3521 & 0.0779 \\
\hline 0.100 & 7.18 & 5.0707 & 2.2596 & -0.1858 & 14.2864 & 0.0804 & 4.6547 & 2.2162 & -0.1805 & 14.3323 & 0.0770 \\
\hline 0.150 & 7.14 & 3.9557 & 2.3941 & -0.2019 & 14.6266 & 0.0896 & 3.7754 & 2.2713 & -0.1861 & 14.4085 & 0.0753 \\
\hline 0.200 & 6.86 & 3.4416 & 2.4461 & -0.2113 & 13.5512 & 0.0913 & 3.3398 & 2.2173 & -0.1812 & 14.7961 & 0.0760 \\
\hline 0.300 & 6.93 & 2.9739 & 2.1595 & -0.1820 & 14.0826 & 0.0767 & 2.8382 & 2.0607 & -0.1685 & 14.2583 & 0.0670 \\
\hline 0.400 & 5.00 & 2.8620 & 2.6300 & -0.2450 & 12.5800 & 0.1109 & 2.5330 & 2.0270 & -0.1670 & 14.3400 & 0.0653 \\
\hline 0.500 & 7.86 & 2.4832 & 1.7376 & -0.1303 & 14.1909 & 0.0504 & 2.4768 & 1.7511 & -0.1328 & 14.2592 & 0.0530 \\
\hline 0.600 & 5.93 & 2.5433 & 1.8708 & -0.1540 & 13.6057 & 0.0636 & 2.3964 & 1.6666 & -0.1196 & 14.9641 & 0.0460 \\
\hline 0.800 & 7.93 & 2.1886 & 1.5351 & -0.1036 & 14.2265 & 0.0431 & 2.2132 & 1.5206 & -0.1003 & 14.3684 & 0.0398 \\
\hline 1.000 & 8.00 & 2.0980 & 1.4180 & -0.0840 & 14.3500 & 0.0333 & 2.1040 & 1.4270 & -0.0860 & 14.2000 & 0.0347 \\
\hline 1.500 & 4.94 & 2.0791 & 1.3392 & -0.0851 & 13.7372 & 0.0486 & 1.9349 & 1.2856 & -0.0622 & 14.3083 & 0.0280 \\
\hline 2.000 & 6.10 & 1.8939 & 1.1904 & -0.0436 & 13.9859 & 0.0203 & 1.8422 & 1.1679 & -0.0373 & 14.4708 & 0.0146 \\
\hline 3.000 & 6.13 & 1.7474 & 1.0584 & -0.0140 & 12.2459 & 0.0058 & 1.7141 & 1.0509 & -0.0113 & 14.2474 & 0.0029 \\
\hline 4.000 & 6.10 & 1.6507 & 0.9863 & 0.0040 & 24.6045 & -0.0076 & 1.6263 & 0.9890 & 0.0034 & 12.9755 & -0.0025 \\
\hline 5.000 & 6.08 & 1.7412 & 0.9391 & 0.0171 & 14.4253 & -0.0111 & 1.5642 & 0.9457 & 0.0146 & 14.9250 & -0.0074 \\
\hline 6.000 & 6.06 & 1.5245 & 0.9075 & 0.0263 & 14.2360 & -0.0151 & 1.5174 & 0.9023 & 0.0292 & 12.6626 & -0.0184 \\
\hline 8.000 & 6.07 & 1.4387 & 0.8695 & 0.0376 & 16.6652 & -0.0336 & 1.4300 & 0.8828 & 0.0335 & 12.1046 & -0.0164 \\
\hline 10.000 & 6.07 & 1.3721 & 0.8563 & 0.0416 & 12.4869 & -0.0209 & 1.3770 & 0.8598 & 0.0399 & 14.3207 & -0.0221 \\
\hline 15.000 & 6.07 & 1.2756 & 0.8410 & 0.0425 & 15.2524 & -0.0299 & 1.2822 & 0.8381 & 0.0472 & 15.8404 & -0.0326 \\
\hline
\end{tabular}


Table 3 (EBF and EABF) G-P fitting coefficients (b, c, a, $X_{k}$ and d) of Influenza (CAS Number 141368-69-6).

\begin{tabular}{|c|c|c|c|c|c|c|c|c|c|c|c|}
\hline \multirow{2}{*}{$\begin{array}{c}\mathbf{E} \\
(\mathrm{MeV})\end{array}$} & \multirow[t]{2}{*}{$\mathbf{Z}_{\mathrm{eq}}$} & \multicolumn{5}{|c|}{ EBF } & \multicolumn{5}{|c|}{ 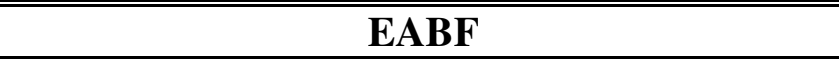 } \\
\hline & & b & $\bar{c}$ & $\bar{a}$ & $\overline{X_{k}}$ & d & $\bar{b}$ & $\bar{c}$ & $\mathbf{a}$ & $\overline{\mathbf{X}_{\mathrm{k}}}$ & $\bar{d}$ \\
\hline 0.015 & 6.33 & 1.3329 & 0.5167 & 0.1547 & 14.2960 & -0.0767 & 1.3415 & 0.5117 & 0.1565 & 14.4724 & -0.0779 \\
\hline 0.020 & 6.34 & 1.7562 & 0.6792 & 0.0974 & 16.0023 & -0.0481 & 1.7767 & 0.6808 & 0.0970 & 15.8959 & -0.0471 \\
\hline 0.030 & 6.35 & 3.1842 & 1.0388 & 0.0024 & 13.8812 & -0.0091 & 3.3530 & 1.0392 & 0.0020 & 13.8479 & -0.0083 \\
\hline 0.040 & 6.36 & 4.7654 & 1.5697 & -0.1004 & 13.7859 & 0.0428 & 4.6603 & 1.5608 & -0.0984 & 13.9872 & 0.0410 \\
\hline 0.050 & 6.34 & 6.1036 & 1.9389 & -0.1494 & 14.1629 & 0.0646 & 5.3411 & 1.9085 & -0.1448 & 14.2921 & 0.0613 \\
\hline 0.060 & 6.36 & 6.5665 & 2.2143 & -0.1814 & 14.2086 & 0.0816 & 5.3181 & 2.1478 & -0.1728 & 14.3235 & 0.0752 \\
\hline 0.080 & 6.34 & 6.2863 & 2.5445 & -0.2151 & 14.0306 & 0.0960 & 4.8803 & 2.3936 & -0.1975 & 14.3144 & 0.0829 \\
\hline 0.100 & 6.33 & 5.6521 & 2.6451 & -0.2223 & 14.3965 & 0.0969 & 4.4292 & 2.4434 & -0.1999 & 14.7405 & 0.0812 \\
\hline 0.150 & 6.36 & 4.2276 & 2.7118 & -0.2322 & 14.1288 & 0.1021 & 3.6074 & 2.4193 & -0.1990 & 14.7547 & 0.0784 \\
\hline 0.200 & 6.86 & 3.4430 & 2.4473 & -0.2114 & 13.5582 & 0.0914 & 3.3390 & 2.2180 & -0.1813 & 14.7959 & 0.0760 \\
\hline 0.300 & 4.00 & 3.4480 & 3.4420 & -0.3040 & 14.2000 & 0.1422 & 2.7220 & 2.1830 & -0.1750 & 15.8800 & 0.0592 \\
\hline 0.400 & 5.00 & 2.8620 & 2.6300 & -0.2450 & 12.5800 & 0.1109 & 2.5330 & 2.0270 & -0.1670 & 14.3400 & 0.0653 \\
\hline 0.500 & 5.93 & 2.6585 & 2.0350 & -0.1744 & 14.0904 & 0.0811 & 2.4543 & 1.8049 & -0.1387 & 15.9498 & 0.0617 \\
\hline 0.600 & 5.93 & 2.5433 & 1.8715 & -0.1541 & 13.6059 & 0.0637 & 2.3963 & 1.6668 & -0.1197 & 14.9635 & 0.0460 \\
\hline 0.800 & 7.00 & 2.2410 & 1.5800 & -0.1120 & 14.0300 & 0.0484 & 2.2020 & 1.5440 & -0.1050 & 14.2000 & 0.0434 \\
\hline 1.000 & 8.00 & 2.0980 & 1.4180 & -0.0840 & 14.3500 & 0.0333 & 2.1040 & 1.4270 & -0.0860 & 14.2000 & 0.0347 \\
\hline 1.500 & 6.35 & 1.9950 & 1.2957 & -0.0649 & 14.1070 & 0.0300 & 1.9373 & 1.2757 & -0.0600 & 14.3235 & 0.0264 \\
\hline 2.000 & 5.73 & 1.9126 & 1.1974 & -0.0455 & 14.1052 & 0.0224 & 1.8404 & 1.1711 & -0.0383 & 14.4276 & 0.0156 \\
\hline 3.000 & 5.73 & 1.7603 & 1.0608 & -0.0150 & 12.1702 & 0.0072 & 1.7150 & 1.0510 & -0.0115 & 14.1170 & 0.0034 \\
\hline 4.000 & 5.74 & 1.6605 & 0.9841 & 0.0045 & 23.5781 & -0.0072 & 1.6270 & 0.9890 & 0.0032 & 13.3379 & -0.0024 \\
\hline 5.000 & 5.72 & 1.7162 & 0.9374 & 0.0173 & 14.6163 & -0.0106 & 1.5658 & 0.9447 & 0.0148 & 14.6185 & -0.0076 \\
\hline 6.000 & 5.73 & 1.5291 & 0.9070 & 0.0260 & 14.5040 & -0.0147 & 1.5205 & 0.9008 & 0.0292 & 12.5608 & -0.0175 \\
\hline 8.000 & 5.74 & 1.4417 & 0.8692 & 0.0373 & 16.3783 & -0.0315 & 1.4350 & 0.8773 & 0.0354 & 11.8793 & -0.0175 \\
\hline 10.000 & 5.75 & 1.3754 & 0.8545 & 0.0418 & 12.6041 & -0.0201 & 1.3806 & 0.8583 & 0.0400 & 14.3647 & -0.0218 \\
\hline 15.000 & 5.74 & 1.2784 & 0.8384 & 0.0434 & 14.9658 & -0.0283 & 1.2859 & 0.8370 & 0.0470 & 15.4645 & -0.0310 \\
\hline
\end{tabular}


Table 4 (EBF and EABF) G-P fitting coefficients (b, c, a, $X_{k}$ and d) of Covid-19 GlycoProtein 6VSB.

\begin{tabular}{|c|c|c|c|c|c|c|c|c|c|c|c|}
\hline \multirow{2}{*}{$\begin{array}{c}\mathbf{E} \\
(\mathrm{MeV})\end{array}$} & \multirow[t]{2}{*}{$\overline{\mathbf{Z}_{\mathrm{eq}}}$} & \multicolumn{5}{|c|}{ EBF } & \multicolumn{5}{|c|}{$\overline{\text { EABF }}$} \\
\hline & & $\mathbf{b}$ & c & $\mathbf{a}$ & $\overline{X_{k}}$ & $\bar{d}$ & $\bar{b}$ & $\bar{c}$ & $\bar{a}$ & $\overline{X_{k}}$ & d \\
\hline 0.015 & 6.67 & 1.2820 & 0.4952 & 0.1631 & 14.2826 & -0.0814 & 1.2872 & 0.4979 & 0.1598 & 14.5226 & -0.0782 \\
\hline 0.020 & 6.70 & 1.6373 & 0.6272 & 0.1156 & 15.4434 & -0.0565 & 1.6553 & 0.6235 & 0.1176 & 15.3406 & -0.0577 \\
\hline 0.030 & 6.74 & 2.8390 & 0.9182 & 0.0334 & 15.0022 & -0.0279 & 2.9601 & 0.9189 & 0.0327 & 15.1977 & -0.0261 \\
\hline 0.040 & 6.77 & 4.2358 & 1.3821 & -0.0698 & 13.6530 & 0.0275 & 4.2237 & 1.3744 & -0.0678 & 13.8664 & 0.0255 \\
\hline 0.050 & 6.79 & 5.3757 & 1.7345 & -0.1245 & 13.9490 & 0.0539 & 5.0354 & 1.7181 & -0.1217 & 14.0650 & 0.0517 \\
\hline 0.060 & 6.80 & 5.8337 & 2.0220 & -0.1624 & 13.8420 & 0.0745 & 5.1974 & 1.9891 & -0.1577 & 13.9396 & 0.0708 \\
\hline 0.080 & 6.80 & 5.6857 & 2.3606 & -0.2003 & 13.5170 & 0.0912 & 4.9033 & 2.2751 & -0.1894 & 13.6926 & 0.0826 \\
\hline 0.100 & 6.82 & 5.3263 & 2.4000 & -0.1993 & 14.3917 & 0.0863 & 4.5992 & 2.3000 & -0.1875 & 14.5494 & 0.0781 \\
\hline 0.150 & 6.78 & 4.0538 & 2.5372 & -0.2165 & 14.1161 & 0.0968 & 3.7029 & 2.3381 & -0.1923 & 14.5221 & 0.0771 \\
\hline 0.200 & 6.83 & 3.4524 & 2.4549 & -0.2120 & 13.6046 & 0.0918 & 3.3340 & 2.2225 & -0.1817 & 14.7951 & 0.0760 \\
\hline 0.300 & 4.00 & 3.4480 & 3.4420 & -0.3040 & 14.2000 & 0.1422 & 2.7220 & 2.1830 & -0.1750 & 15.8800 & 0.0592 \\
\hline 0.400 & 5.00 & 2.8620 & 2.6300 & -0.2450 & 12.5800 & 0.1109 & 2.5330 & 2.0270 & -0.1670 & 14.3400 & 0.0653 \\
\hline 0.500 & 5.91 & 2.6587 & 2.0398 & -0.1750 & 14.0706 & 0.0815 & 2.4539 & 1.8057 & -0.1388 & 15.9298 & 0.0617 \\
\hline 0.600 & 5.91 & 2.5433 & 1.8755 & -0.1547 & 13.6071 & 0.0642 & 2.3958 & 1.6675 & -0.1198 & 14.9602 & 0.0461 \\
\hline 0.800 & 7.00 & 2.2410 & 1.5800 & -0.1120 & 14.0300 & 0.0484 & 2.2020 & 1.5440 & -0.1050 & 14.2000 & 0.0434 \\
\hline 1.000 & 8.00 & 2.0980 & 1.4180 & -0.0840 & 14.3500 & 0.0333 & 2.1040 & 1.4270 & -0.0860 & 14.2000 & 0.0347 \\
\hline 1.500 & 6.23 & 2.0006 & 1.3011 & -0.0663 & 13.9605 & 0.0311 & 1.9386 & 1.2755 & -0.0600 & 14.3117 & 0.0265 \\
\hline 2.000 & 5.63 & 1.9178 & 1.1993 & -0.0461 & 14.1461 & 0.0231 & 1.8395 & 1.1725 & -0.0387 & 14.3913 & 0.0160 \\
\hline 3.000 & 5.77 & 1.7592 & 1.0604 & -0.0149 & 12.1458 & 0.0070 & 1.7150 & 1.0509 & -0.0114 & 14.1689 & 0.0033 \\
\hline 4.000 & 5.76 & 1.6599 & 0.9842 & 0.0044 & 23.7502 & -0.0072 & 1.6269 & 0.9891 & 0.0032 & 13.2947 & -0.0023 \\
\hline 5.000 & 5.77 & 1.7233 & 0.9377 & 0.0172 & 14.5959 & -0.0107 & 1.5655 & 0.9451 & 0.0146 & 14.6942 & -0.0075 \\
\hline 6.000 & 5.74 & 1.5288 & 0.9070 & 0.0260 & 14.4940 & -0.0147 & 1.5204 & 0.9007 & 0.0293 & 12.5462 & -0.0176 \\
\hline 8.000 & 5.77 & 1.4415 & 0.8691 & 0.0374 & 16.4481 & -0.0318 & 1.4345 & 0.8779 & 0.0352 & 11.9022 & -0.0174 \\
\hline 10.000 & 5.76 & 1.3752 & 0.8546 & 0.0418 & 12.5868 & -0.0202 & 1.3804 & 0.8583 & 0.0400 & 14.3617 & -0.0218 \\
\hline 15.000 & 5.75 & 1.2783 & 0.8385 & 0.0434 & 14.9796 & -0.0284 & 1.2857 & 0.8371 & 0.0470 & 15.4846 & -0.0311 \\
\hline
\end{tabular}




\section{Figures}

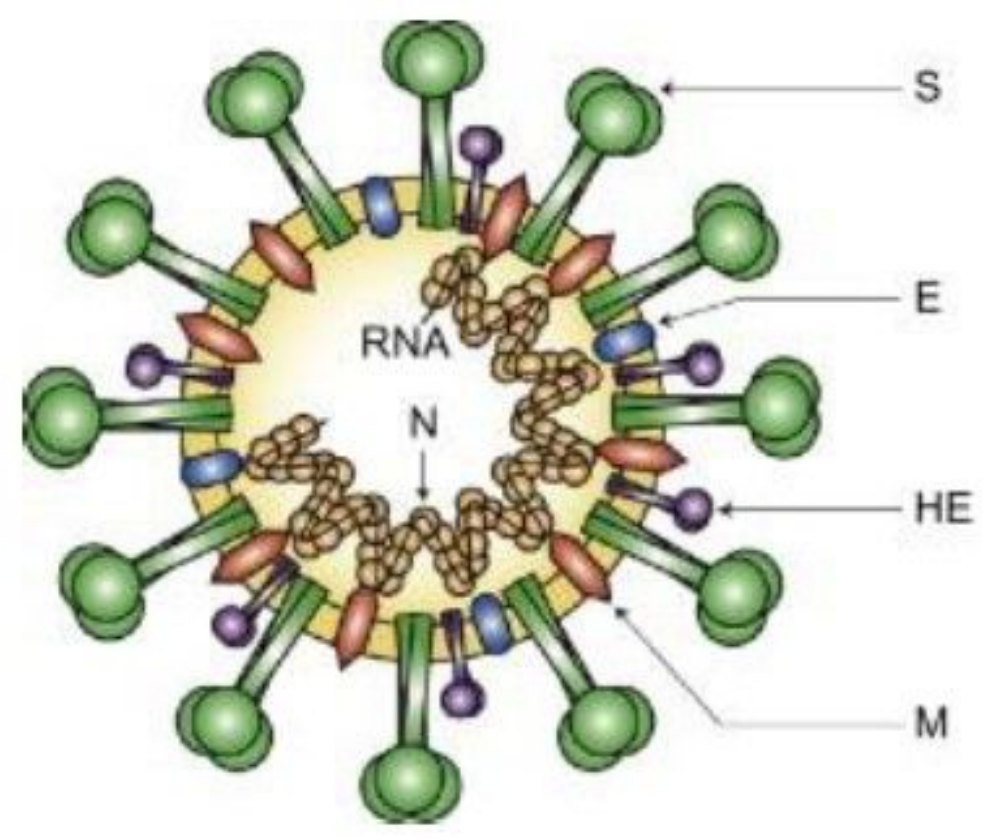

Figure 1

Coronavirus structure with structural proteins. N: Nucleocapsid protein; S: Spike protein, M: Membrane protein, HE: Hemagglutinin-Esterase and E: Envelope protein 


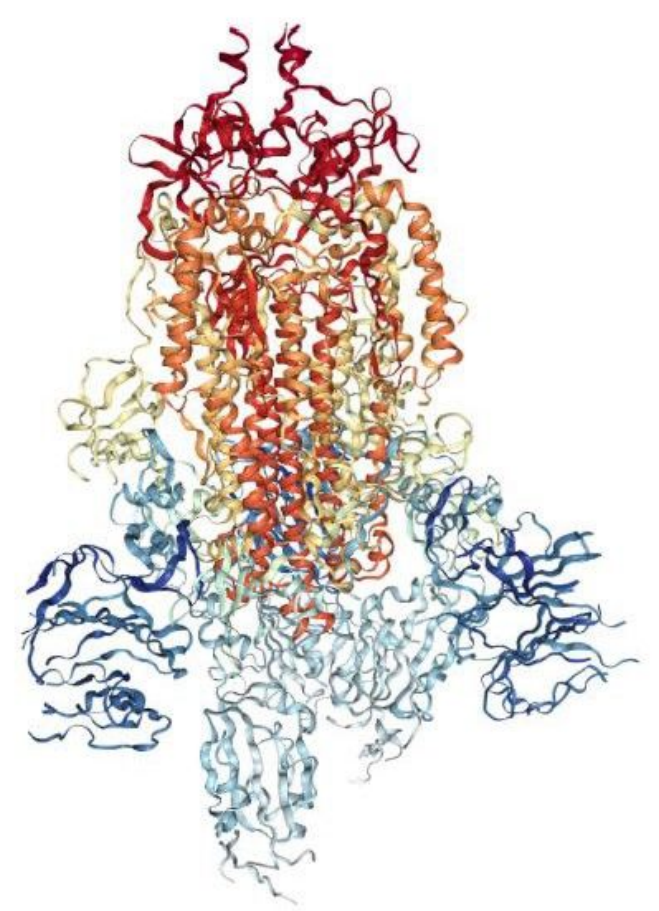

(a)

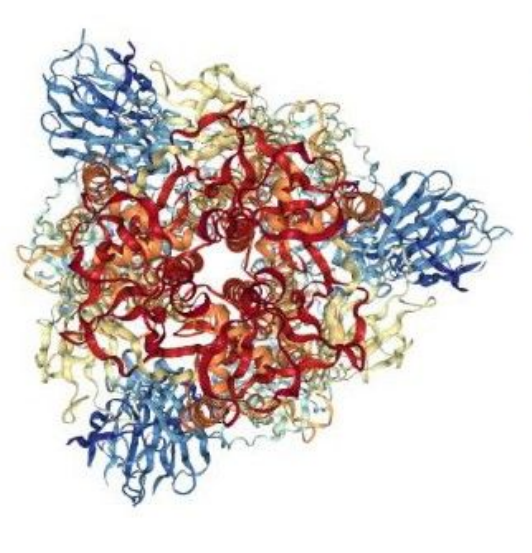

(b)

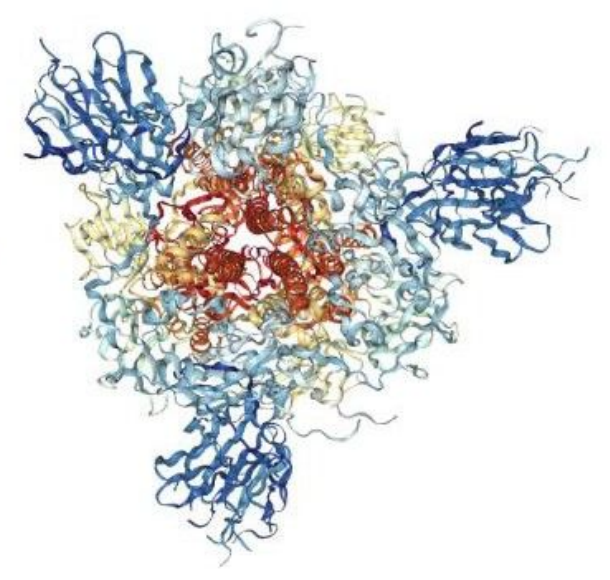

\section{Figure 2}

The Structure of Human Coronavirus GlycoProtein (6VSB) (a) side view (b) top view (c) bottom view

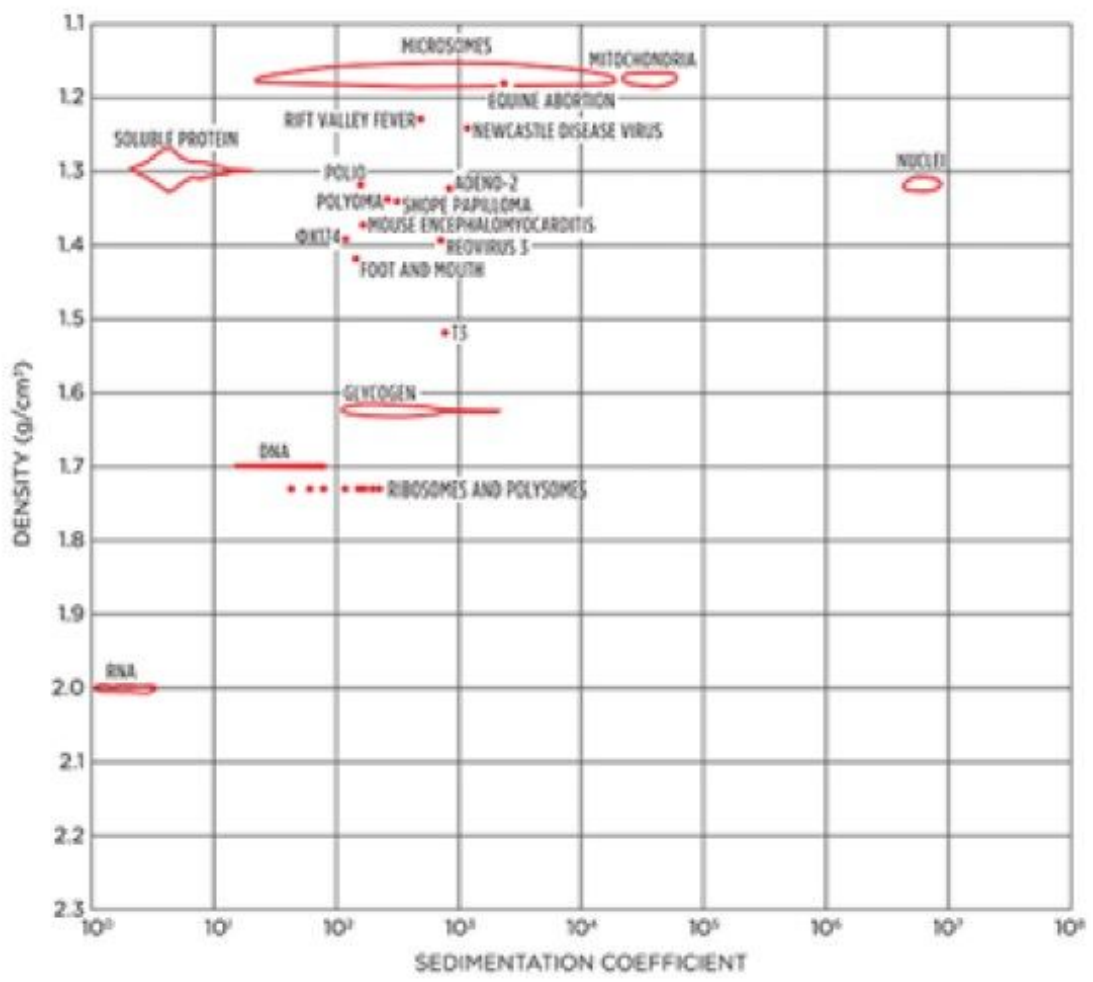


Figure 3

Virus/Cell Organelle densities and S values

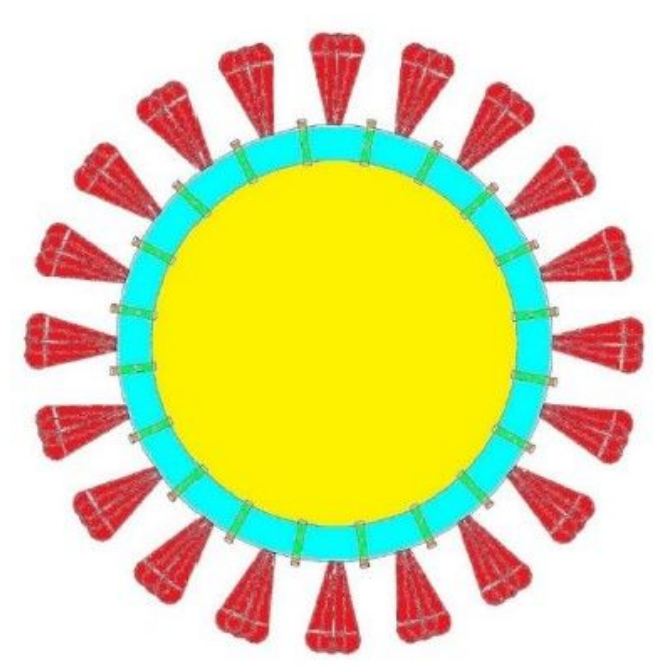

(a)

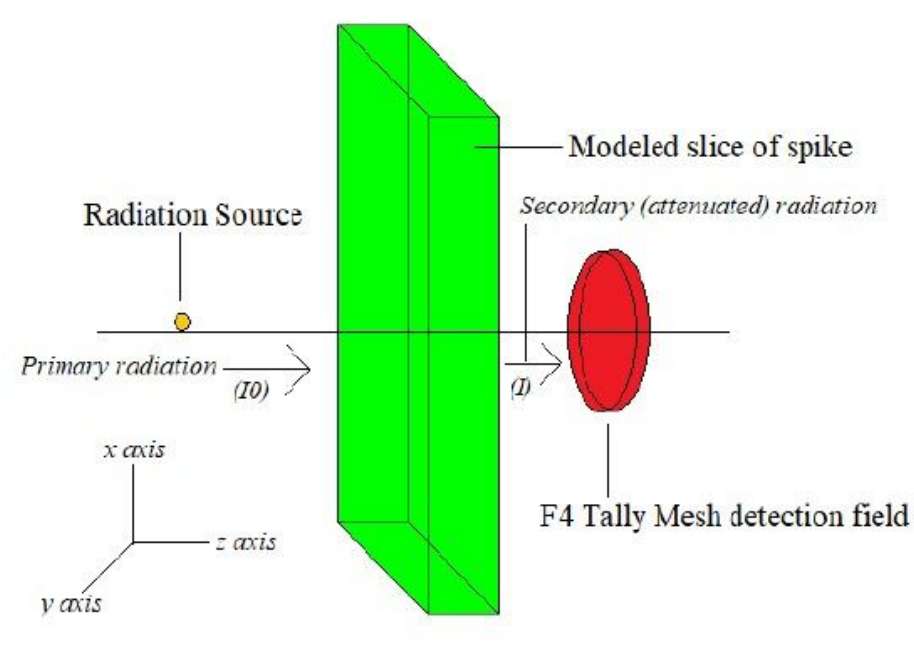

(b)

Figure 4

(a) Axial view of modeled virus structure in MCNPX code (b) Radiation transmission setup for calculation of attenuation coefficients 


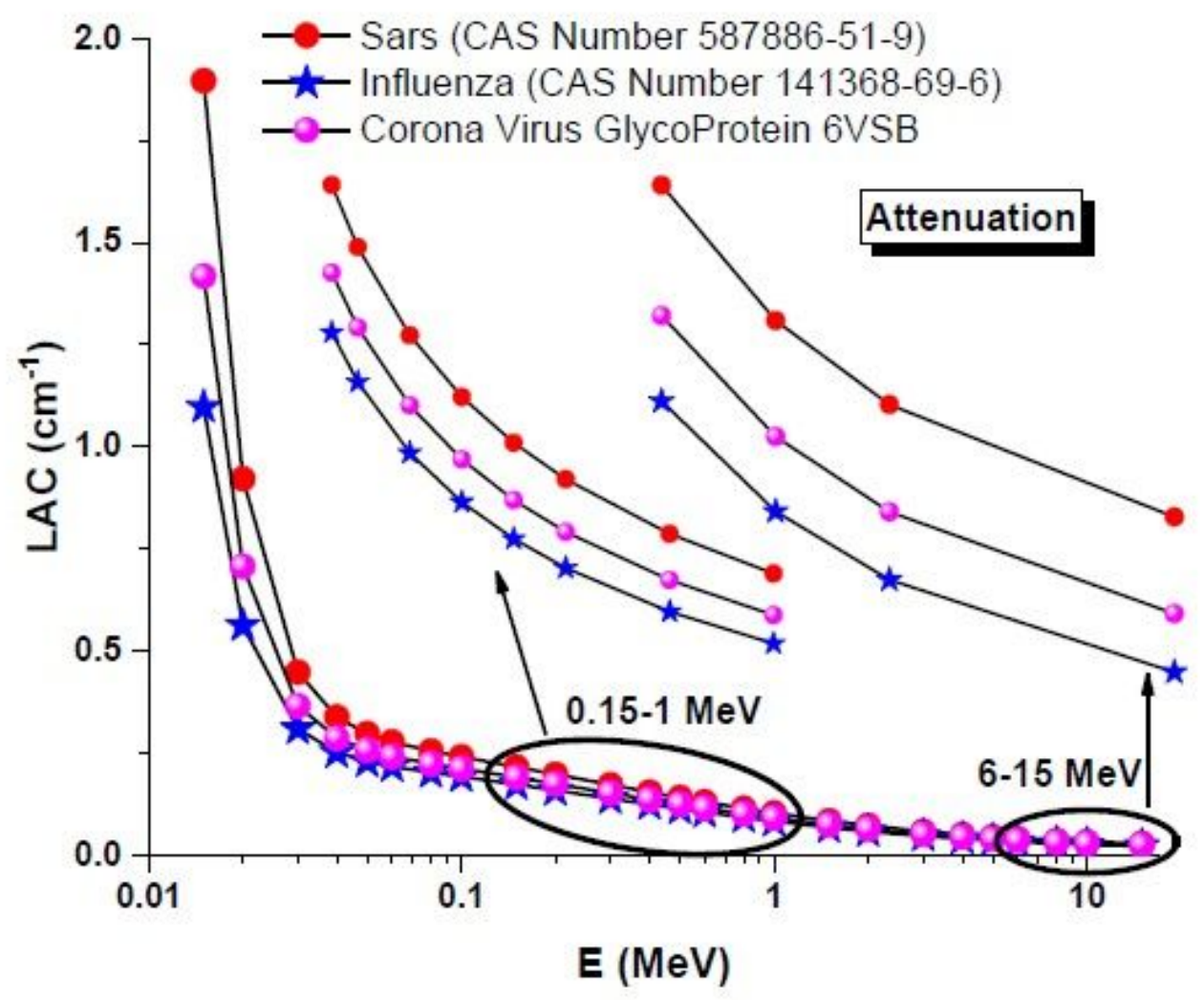

Figure 5

Linear attenuation coefficient (LAC) values as a function of photon energy of Sars (CAS Number 58788651-9), Influenza (CAS Number 141368-69-6) and Covid-19 GlycoProtein 6VSB. 


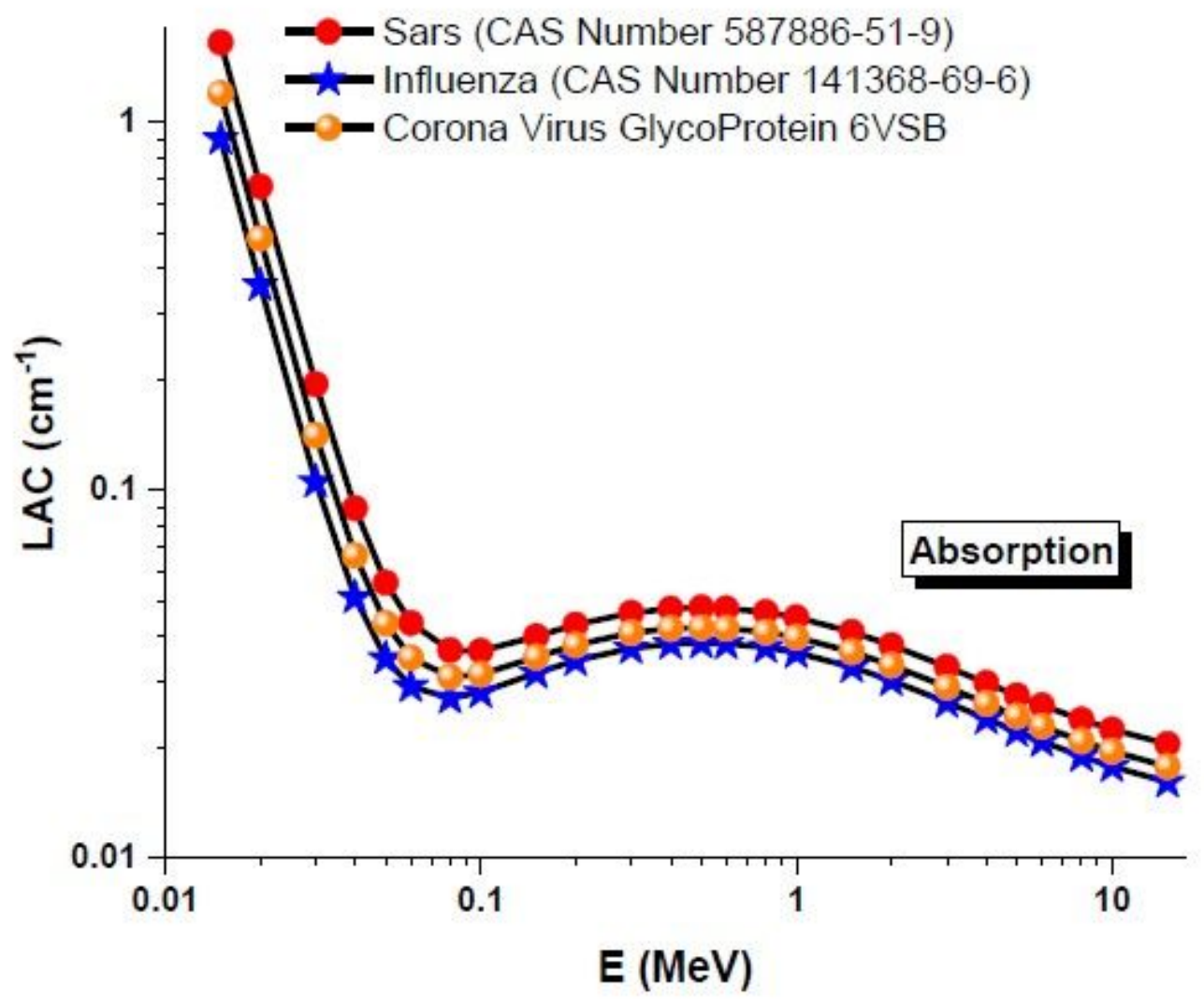

Figure 6

linear absorption coefficient (LAC) values as a function of photon energy of Sars (CAS Number 58788651-9), Influenza (CAS Number 141368-69-6) and Covid-19 GlycoProtein 6VSB. 


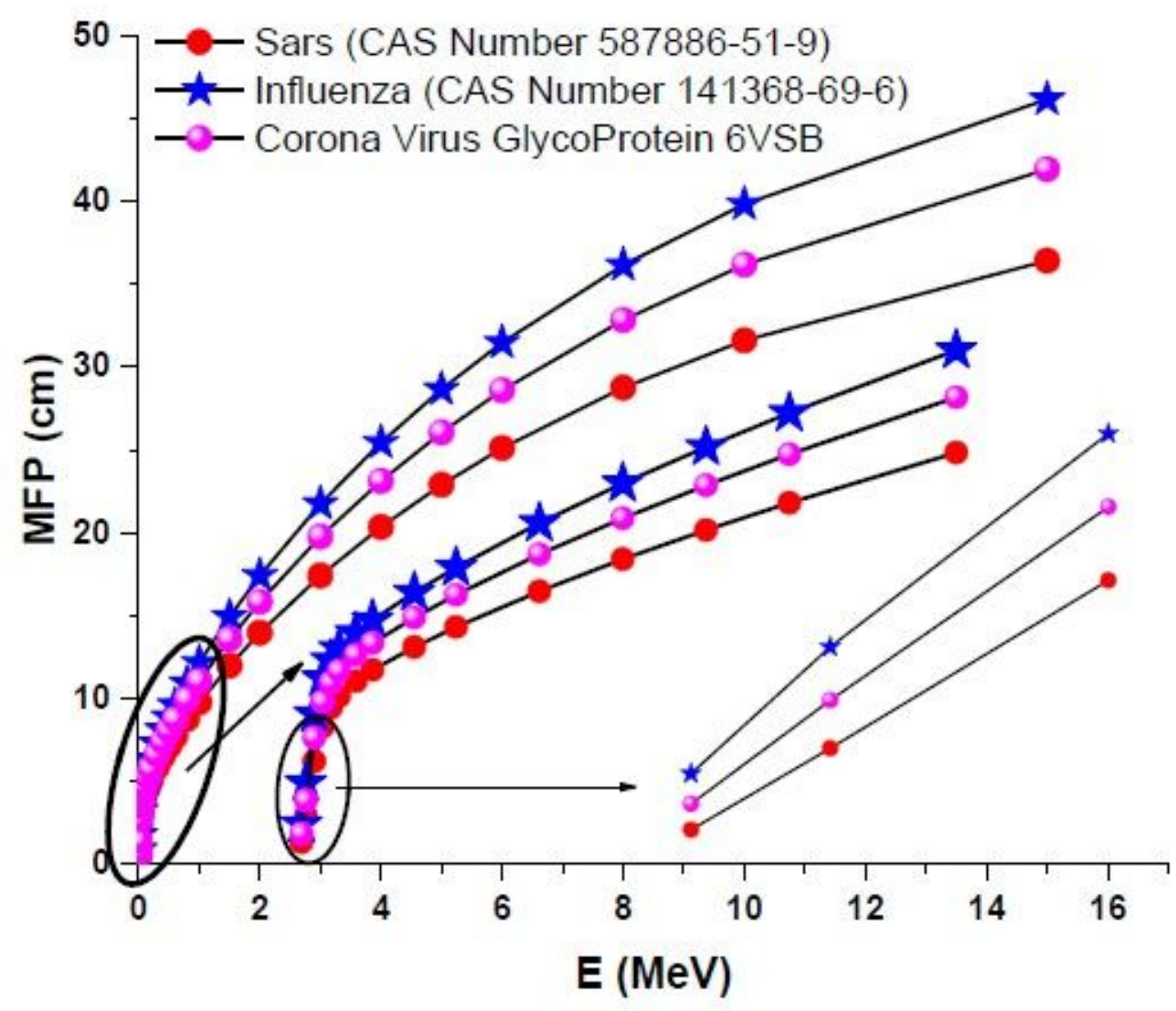

Figure 7

Mean free path (MFP) values as a function of photon energy of Sars (CAS Number 587886-51-9), Influenza (CAS Number 141368-69-6) and Covid-19 GlycoProtein 6VSB. 


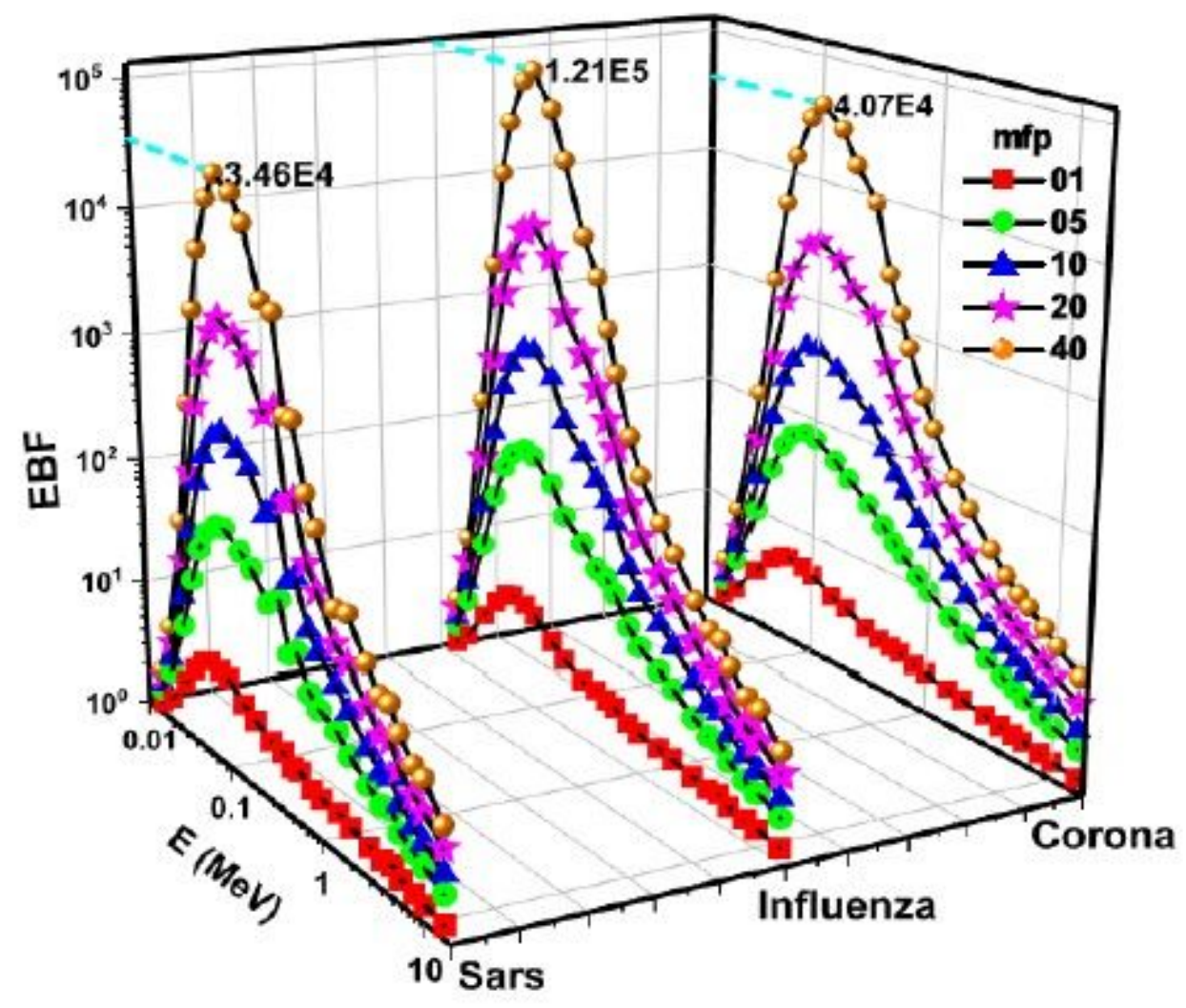

Figure 8

Exposure buildup factor (EBF) values as a function of photon energy of Sars (CAS Number 587886-51-9), Influenza (CAS Number 141368-69-6) and Covid-19 GlycoProtein 6VSB. 


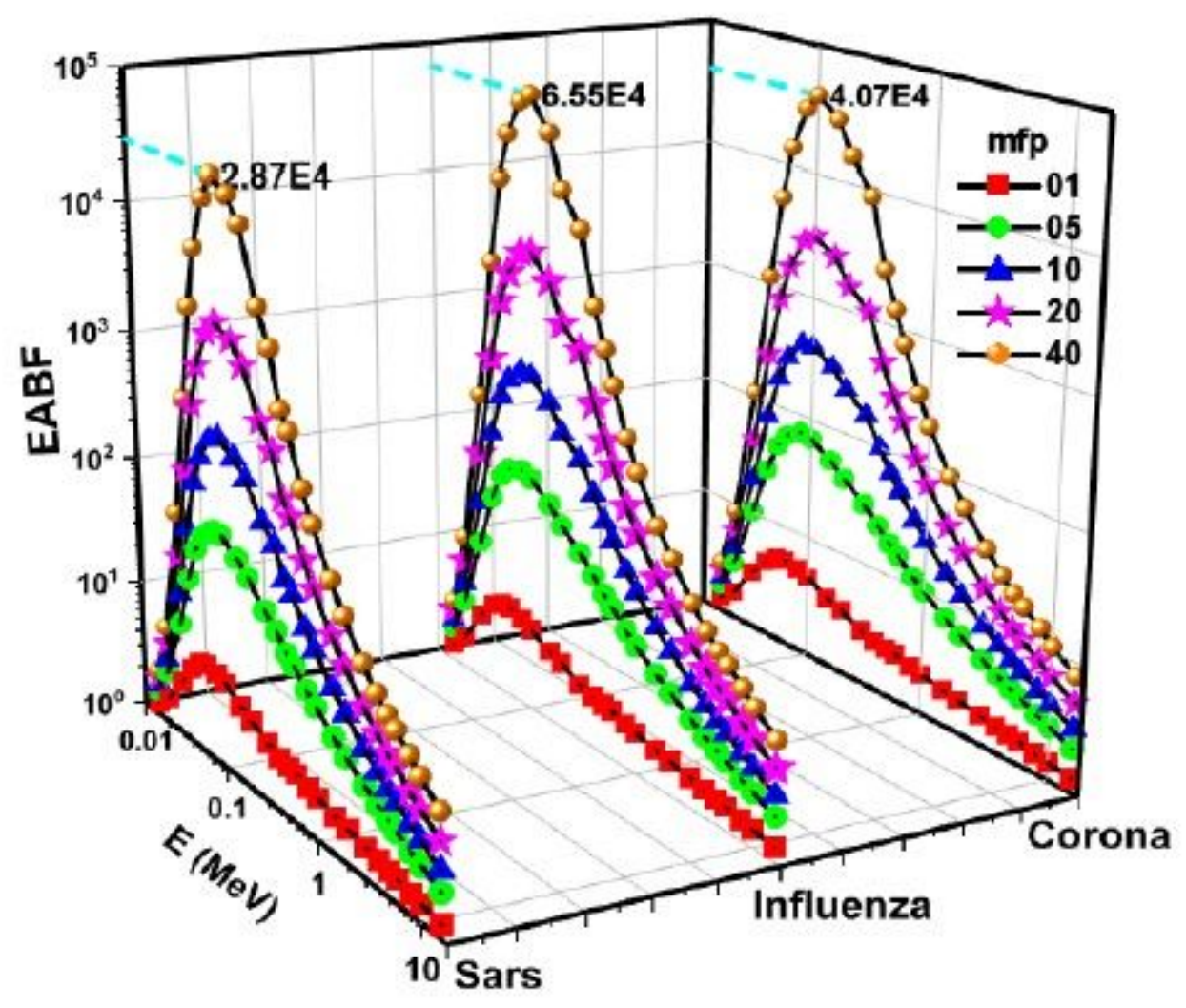

Figure 9

Energy absorption buildup factor (EABF) values as a function of photon energy of Sars (CAS Number 587886-51-9), Influenza (CAS Number 141368-69-6) and Covid-19 GlycoProtein 6VSB. 

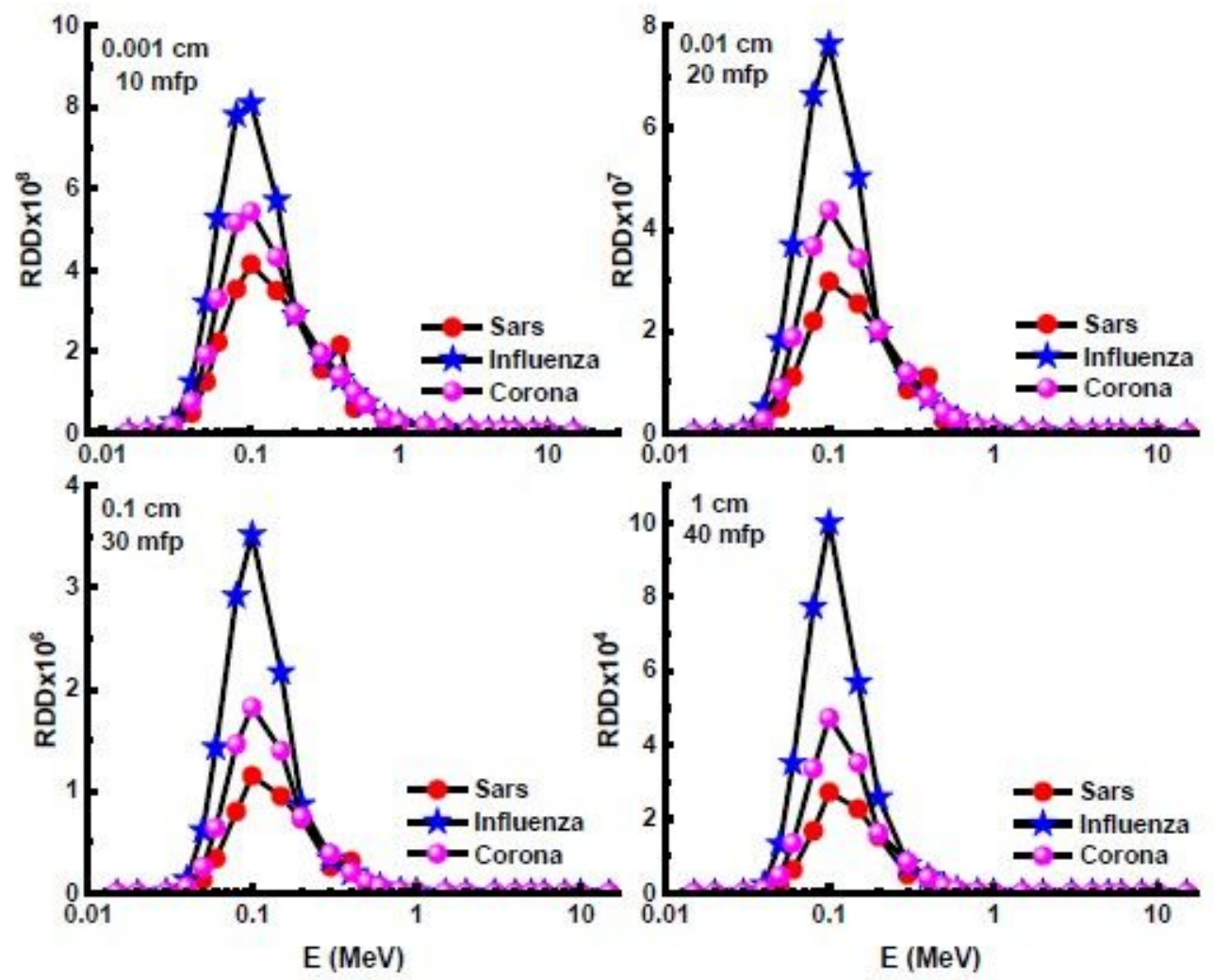

Figure 10

Relative dose distribution (RDD) values as a function of photon energy at different penetration depth (mfp) and distances (cm) of Sars (CAS Number 587886-51-9), Influenza (CAS Number 141368-69-6) and Covid-19 GlycoProtein 6VSB. 

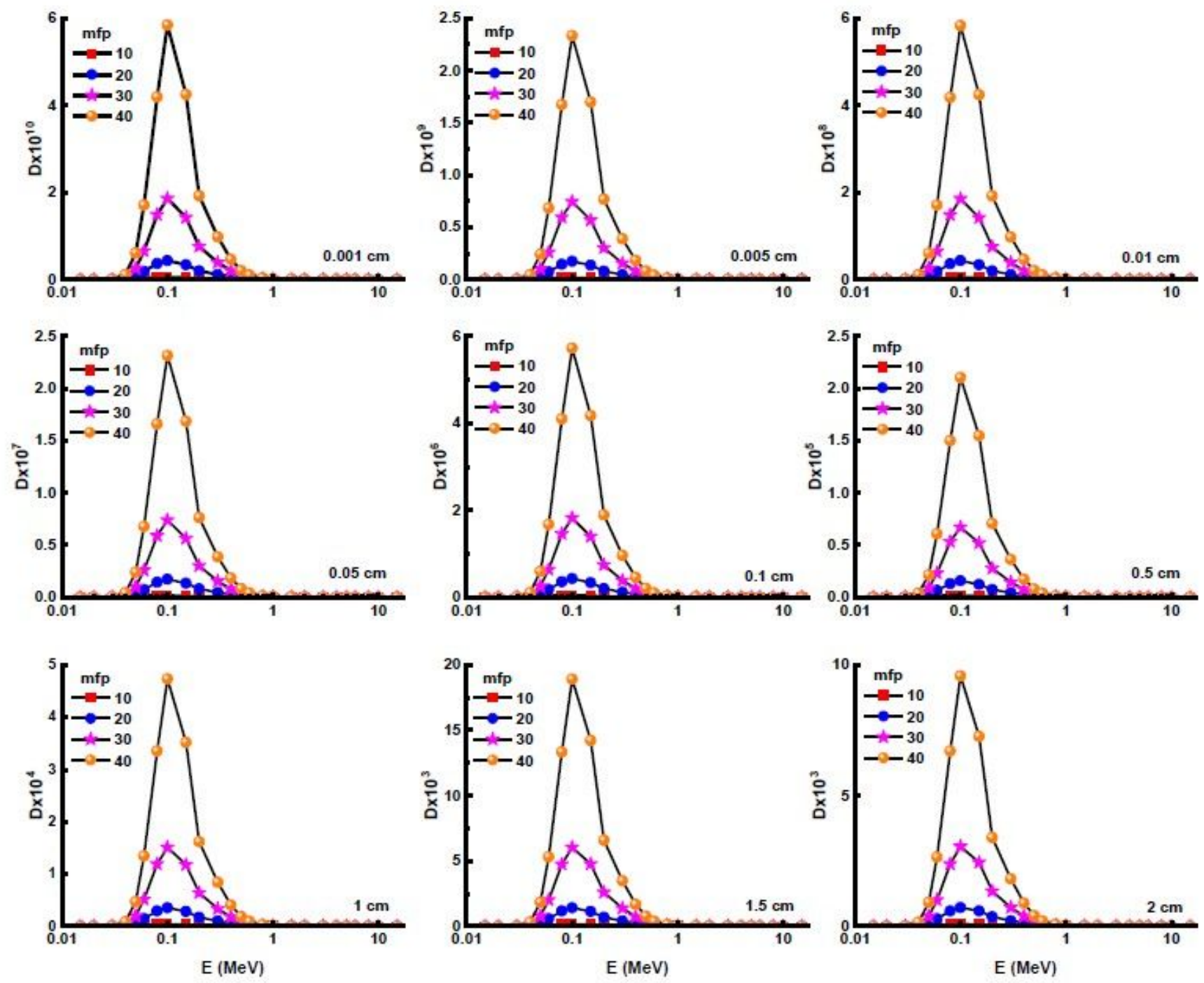

Figure 11

Relative dose distribution values as a function of photon energy at different penetration depth (mfp) and distance $(\mathrm{cm})$ of Covid-19 GlycoProtein 6VSB. 